\title{
Norepinephrine transporter defects lead to sympathetic hyperactivity in stem cell and mouse models of Familial Dysautonomia
}

Hsueh Fu Wu

University of Georgia

Wenxin Yu

University of Georgia

Joeseph Carey

Montana State University, Bozeman

Frances Lefcort

Montana State University

Hongxiang Liu

University of Georgia

Nadja Zeltner ( $\square$ nadja.zeltner@uga.edu )

Universtiy of Georgia https://orcid.org/0000-0002-2978-2507

\section{Article}

Keywords: Familial Dysautonomia (FD), human pluripotent stem cell (hPSC) technology, sympathetic neuron (symN)-specific disease mechanisms

Posted Date: November 24th, 2021

DOI: https://doi.org/10.21203/rs.3.rs-1024649/v1

License: (9) This work is licensed under a Creative Commons Attribution 4.0 International License.

Read Full License

Version of Record: A version of this preprint was published at Nature Communications on November 17th, 2022. See the published version at https://doi.org/10.1038/s41467-022-34811-7. 


\section{Abstract}

Familial dysautonomia (FD) is a rare neurodevelopmental and neurodegenerative disorder that affects the sympathetic nervous system. Patients harbor a mutation in ELP1 yet, how loss of Elp1 affects the function of symNs remains unresolved. Such an understanding is critical since the most debilitating hallmarks of the disease include cardiovascular instability, dysautonomic crises and renal failure, which all result from dysregulated sympathetic activity. Here, we employ the human pluripotent stem cell (hPSC) technology as a modeling system to understand human, sympathetic neuron (symN)-specific disease mechanisms and provide a platform for drug testing and discovery. We show that FD symNs are intrinsically hyperactive in vitro, in co-cultures with cardiomyocyte target tissue and in FD animal models. We show that ELP1-rescued isogenic lines remain hyperactive, suggesting a different/additional disease mechanism. Accordingly, we report decreased intracellular norepinephrine (NE) levels, decreased NE reuptake via NET and excessive extracellular NE in FD symNs. Finally, we performed a mini drug screen showing that current and new candidate drugs were able to lower hyperactivity. These findings may have implications for other peripheral nervous system disorders. Our drug screening platform may allow future drug testing and discovery for such disorders.

\section{Introduction}

Familial Dysautonomia (FD) is a complicated, early-onset, genetic disorder that mainly affects the development of the sensory and sympathetic nervous system (SNS) within the peripheral nervous system (PNS), leading to reduced numbers of these neurons. It also leads to neurodegeneration of these cells. $99.5 \%$ of all FD patients harbor a nonsense RNA splicing mutation in the gene ELP1 (formerly called $I K B K A P^{1}$, which encodes the protein elongator complex protein 1 (elp1). Elp1 is the structural component of the elongator complex, which is directly involved in tRNA modification ${ }^{2}$ and is essential for normal translation, and thus can be found in most cells of the body. While FD patients also suffer deficits in the central nervous system (CNS, particularly the eye), due to the critical role of the autonomic nervous system (ANS) in maintaining homeostasis, the altered function of the ANS is the most life-threatening system in FD. It was proposed that ELP1 defects mainly affect particularly long transcripts with an AA nucleotide bias and that many PNS critical genes fall into this category ${ }^{3}$. Interestingly, FD patients vary in the severity of their clinical phenotype, which raises the question of whether modifier genes exist. In fact, it's been shown that FD patients with the most severe symptoms harbor a modifier mutation in the gene $\angle A M B 4$, encoding lamininb $4{ }^{4}$. FD patients suffer from a variety of symptoms, including difficulties swallowing and regulating heart rate and blood pressure ${ }^{5}$. Dysautonomic/adrenergic crises are particularly disabling episodes of tachycardia, arterial hypertension, nausea/vomiting/retching and behavioral changes reminiscent of anxiety attacks ${ }^{6}$. Crisis can be frequent (daily), increase with age ${ }^{7}$, lead to hospitalization and contribute significantly to mortality ${ }^{8}$. Interestingly, crises are triggered by emotional or physical stress and associated with anxiety that can escalate into phobias ${ }^{9}$. Uncontrollable sympathetic neuron (symN) over-stimulation and associated prolonged catecholamine secretion into the blood stream caused by afferent baroreflex failure has been suggested as the mechanisms mediating FD 
crisis $^{10}$. This indeed explains the increase in blood pressure during crisis. Nevertheless, the fact that nonFD patients with baroreflex failure mainly suffer from unstable blood pressure, but do not experience many of the other aspects of the FD dysautonomic crisis, suggests that the loss of central autonomic control and baroreflex defects may not be the only factor that leads to the crisis. For example, vomiting/retching episodes, characteristic for FD dysautonomic crisis, do not occur in baroreflex failure patients. In FD, it was suggested that vomiting/retching is a result of excessive dopamine (DA) in the blood stream ${ }^{11}$. Therefore, we hypothesize that intrinsic factors within symNs in FD contribute to triggering dysautonomic crisis.

Reduced symN numbers and overall sympathetic ganglia size reduction has been reported in FD patients $^{12}$ and in FD mouse models ${ }^{13}$. Such observations are based on both developmental defects ${ }^{13}$ and degeneration ${ }^{12,13}$. While several studies in mouse neurons have revealed that neurons in FD have impairments in mitochondrial function ${ }^{14}$, elevated reactive oxygen species and cytoskeleton issues ${ }^{15}$; that their proteome and transcriptome is affected ${ }^{3}$, and they have faulty NGF retrograde transport and signaling ${ }^{16}$, no study on neuronal activity has been reported, particularly not in human cells. Loss, hypoor hyperactivity of symNs leads to a variety of human disorders and intrinsic neural hyperactivity has been proposed as the cause of dysfunction and ensuing degeneration in several disorders, including amyotrophic lateral sclerosis (ALS) $)^{17}$ and Alzheimer's disease ${ }^{18}$. Hyperactivity is further linked with aberrant calcium homeostasis ${ }^{19}$ and norepinephrine (NE) levels ${ }^{20}$. Therefore, we aim to investigate symN-specific phenotypes in FD, with the long-term goal to provide knowledge for drug development for FD as well as other symN disorders.

Assessments of symN function in the clinic are based on indirect measures, such as blood pressure, heart rate and blood glucose level ${ }^{21}$, and the lack of availability of primary sympathetic tissue for research makes it challenging to address neural intrinsic dysfunctions. Thus, it remains to be shown is mechanistic insights into FD gained from mouse studies are present in human cells as well. The human pluripotent stem cell (hPSC) technology is ideal to address such shortcomings, as it allows the generation of near unlimited numbers of human, patient-derived symNs and their observation and manipulation in the dish ${ }^{22}$.

Here, we employ our well-established symN differentiation protocol ${ }^{23}$ to study functional phenotypes in FD symNs, using FD patient- and healthy control-derived hPSCs. We recapitulate developmental defects in the sympathetic lineage and reveal spontaneous, intrinsic hyperactivity of FD symNs. We identify a defective norepinephrine autoregulatory pathway that underlies the hyperactivity phenotype. Lastly, we show that this model system is sensitive in confirming clinical drugs and potential drug compounds that can relieve symN hyperactivity. Together, our results reveal symN hyperactivity as a novel pathology in FD, and we provide a novel, human drug testing and screening platform for symN-modulating compounds.

\section{Results}




\section{Developmental phenotypes in the sympathetic lineage in Familial Dysautonomia}

To model symN defects in FD, we needed an efficient and reproducible differentiation protocol to derive pure symNs from hPSCs. Although there are several published symN differentiation strategies ${ }^{23-27}$, we first further optimized our previous feeder-free, chemically defined protocol (Sup. Fig. 1a). Neural crest cells (NCCs) at day 10 are derived at an efficiency of $>90 \%{ }^{23}$, thus a time-consuming flow cytometry (FACS) purification step can be omitted. SymNs from day 14 on express sympathoblast genes including PHOX2B, ASCL1 and HAND2 (Sup. Fig. 1b) and express HOX genes (HOX 5-9) indicating their trunk-like identity (Sup. Fig. 1C). They display typical PNS ganglia-like morphology (Fig. 1d). Mature marker genes are expressed from day 20 on, on the mRNA and protein level (Sup. Fig. 1e-h), including genes important in symN signal transduction CHRNA3, CHRNB4 and VMAT1, autoregulation $\mathrm{a}_{2} / \beta_{2}$ ARs and NET and NE synthesis/metabolism $T H, D B H, A A A D$ and MOA-A. We also confirmed NE production in symNs (Sup. Fig. $1 \mathrm{~h}$ ). To improve technical ease of the protocol, we show that both human embryonic stem cell (hESC)- and iPSC-derived NCCs can be cryopreserved at day 10, followed by differentiation into symNs, without compromising the neurons quality (Sup. Fig. 1i, j).

Next, we sought to improve the purity of differentiated symNs to prevent possible variations due to contaminating non-neuronal cells during phenotype identification. Our original protocol yielded about $45 \%$ neurons, of which over $90 \%$ were $\mathrm{TH}^{+}$symNs (Sup. Fig. 2a, grey bars). To eliminate non-neuronal lineages, we treated day 20 symNs with aphidicolin (Aphi), a cell cycle inhibitor commonly used in primary peripheral neuron cultures ${ }^{28,29}$, for 10 days. After day 30 , Aphi-treated symNs show significantly improved purity, while neural specificity is not affected (Sup. Fig. 2a, b, red bars). Proliferating Ki $67^{+}$cells and SOX10+ NCCs are dramatically diminished after Aphi treatment (Sup. Fig. 2c). Furthermore, we analyzed electrical activity of symNs after day 20 using multi-electrode array (MEA) (Sup. Fig. 2d). Neural spikes represent functionality and neural bursts represent neuronal maturity, which increases over time (Sup. Fig. 2e, f). Thus, this protocol yields high numbers of pure and functional symNs within a shortened time-period.

With this differentiation protocol in hand, we next aimed to assess phenotypes in the symN lineage in FD. We employed the following hPSC lines: iPSCs from FD patients (iPSC-FD-S2 and iPSC-FD-S3) and healthy control subjects (iPSC-ctrl-C1), and healthy ESCs (hESC-ctrl-H9, Sup. Fig. 3a). These lines were previously established, well characterized and employed in disease modeling ${ }^{4}$. We first assessed disease phenotypes throughout the developmental stages of NCCs, sympathoblasts and symNs in FD (Fig. 1a). NCCs can be identified in condensed dark "ridge" structures (Fig. 1b, arrows), which are $\mathrm{SOX} 10^{+}$and

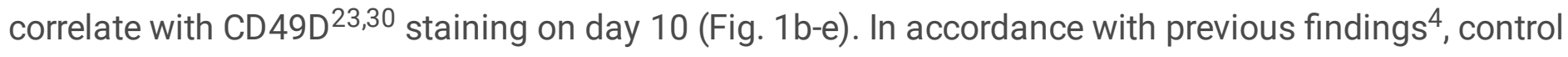
NCCs (iPSC-ctrl-C1 and hESC-ctrl-H9) show higher differentiation efficiency ( 90\%) than FD NCCs (iPSCFD-S2 and iPSC-FD-S3, 30\%) evident by higher SOX10+ NCC ridge coverage (Fig. 1b, c) and higher CD49D expression (by FACS, Fig. 1d, e). Such a ratio of control to FD NCC reduction (90-30\%) correlates 
with the reduction of sympathetic ganglia volume described in FD patients ${ }^{12}$ and mice. From here on, we combined data for iPSC-ctrl-C1 and hESC-ctrl-H9 and call it ctrl, as well as iPSC-FD-S2 and iPSC-FD-S3 and call it FD as indicated in the figure legends. To answer the question, whether in FD the remaining $60 \%$ of cells die or differentiate into another cell type, we assessed overall cell numbers. We found that at day 10 there is no difference in cell numbers (Sup. Fig. 4a), which is corroborated by similar staining for AP2a (Sup. Fig. 4b), suggesting that FD iPSCs may differentiate into other cell types, likely non-neural ectoderm, rather than die. Using RT-qPCR analysis, we found significant expression of SIX $1^{+} / \mathrm{EYA} 1^{+}$ placode contaminant (Sup. Fig. 4c). However, we previously showed that these non-neural cells do not aggregate into neural spheroids or survive ${ }^{23}$, and thus are lost upon further differentiation.

Next, we compared control and FD NCCs during sympathoblast specification in spheroids from day 10-14 (Fig. 1f). Despite replating equal NCC numbers at day 10 in control and FD iPSCs, we observed smaller size as well as lower total cell numbers in FD compared to control spheroids by day 14 (Fig. $1 \mathrm{f}, \mathrm{g}$ ). We also noticed compromised integrity in FD spheroids, forming less compact, smooth and more irregularshaped aggregates (Fig. 1f). Additionally, we compared the survival rate between control and FD cells after dissociation at day 0,10 and 14 and show that survival in FD drops at the sympathoblast stage, where specific symNs are specified (Fig. 1h). Finally, we assessed symN differentiation upon dissociation and equal cell number replating of day 14 sympathoblasts. We found that, at day 30 both control and FD generate normal looking symNs with similar morphology and distribution (Fig. 1i). RT-qPCR analysis showed no significant differences of symN markers (Fig. 1j). SymN morphology was further examined using axonal marker GAP43, dendritic marker MAP2 and synaptic marker synaptophysin, and no difference was found (Fig. 1i, k). Together, our results suggest that in FD both the NCC and sympathoblast numbers are reduced, but the remaining progenitors are still capable of generating symNs. These findings are supported by our previous work ${ }^{4}$ and other's works ${ }^{12,31,32}$.

\section{Sympathetic neurons in Familial Dysautonomia are spontaneously hyperactive}

We next asked if the symNs that do develop in FD are functional. Utilizing MEA, we found that FD symNs are spontaneously hyperactive starting at day 25 (Fig. 2a). To investigate the possibility that the difference found at a single timepoint is because of varying differentiation progression/maturation of each iPSC line, MEA recording was performed in a time course from day 20-60. We found that compared to control, FD symNs are spontaneously hyperactive throughout their maturation and that up to day 60 , control neurons never reach the spike frequency of FD (Fig. 2b), excluding the concern of differential maturation speed. Our finding of hyperactivity in FD symNs was further confirmed by increased expression of c-Fos, a gene that represents symN activity ${ }^{33}$ (Fig. 2c), neuropeptide Y, a factor that will be upregulated following symN activation ${ }^{34}$ (Fig. 2d) and corticotropin releasing hormone receptor 1 and 2 (CRHR1/2), the receptors for stress-induced $\mathrm{CRH}^{35}$ (Fig. 2e), all highlighting increased neuronal activity and heightened stress responsiveness. Additionally, we confirmed FD symN hyperactivity by monitoring spontaneous calcium $\left(\mathrm{Ca}^{2+}\right)$ dynamics. Using the $\mathrm{Ca}^{2+}$ probe Fluo-4, we observed that FD symNs have 
higher $\mathrm{Ca}^{2+}$ influx activity than healthy control symNs during recording ${ }^{36}$ (Fig. 2f), reflecting the hyperactive state in FD symNs. In order to understand whether the hyperactivity phenotype is a PNSspecific feature, we differentiated CNS cortical neurons from control and FD iPSCs using previously described protocols $\mathrm{s}^{37,38}$ (Fig. 2g) and assessed their electric activity in parallel. We did not detect significant differences either in morphology (Fig. 2g) or mean firing rate (Fig. $2 \mathrm{~h}$ ) at or before day 45 . These observations support that symN hyperactivity is a critical PNS phenotype in FD.

We next assessed the effect of hyperactive symNs on regulating their target tissue in FD. We differentiated cardiomyocytes ${ }^{39,40}$ (CMs) from healthy hESC-ctrl-H9 and/or iPSC-ctrl-C1 lines, that started beating at day 7 (Sup. Fig. 5a, Sup. Video 1), fired cardiac action potentials from day 10 on (via MEA, Sup. Fig. 5b) and expressed specific markers $C T n T$ and NKX2.5 at day 15 (Sup. Fig. 5c). To mimic the SNS-cardiac axis, we created a co-culture consisting of day $7 \mathrm{CM}$ precursors and dissociated day 14 symNs in MEA plates (Fig. 2i). Innervation of cardiomyocytes by symNs can be observed one week later (Sup. Fig. 5d). CM beating rate increased when CMs were cultured with conditional medium from symNs, as well as in the co-culture with symNs. It could further be augmented when symNs were stimulated with nicotine (Sup. Fig. 5e), indicating the functionality of the co-culture system. With this powerful tool, we assessed FD symN regulation of its cardiomyocyte target tissue. As shown previously ${ }^{26}$, the co-culture matured and thus increased the beating rate even in control; however, it significantly increased CM beating rate in the FD symN co-culture (Fig. 2j). Together, we show that symNs in FD are spontaneously hyperactive which leads to increased target tissue activation, potentially paralleling FD patients heart rate instability ${ }^{41}$.

\section{Sympathetic neuron hyperactivity is conserved in Familial Dysautonomia mouse models}

Next, we sought to corroborate our findings of symN hyperactivity in FD in vivo. Previous reports in various FD mouse models have shown symN loss at the embryonic stage, smaller sympathetic ganglion size and defective target innervation ${ }^{13,15,42}$. SymN activity, however has not been investigated previously

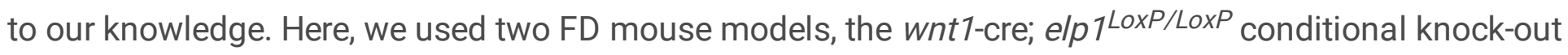
(CKO) model $^{13}$ and the sox 10 -cre; elp $1^{\text {LoxP/LoxP }}$ CKO model ${ }^{43}$, both of which delete elp 1 expression in the neural crest cell lineage (Sup. Fig. 6a). Most FD CKO mice, including ours, are embryonic lethal ${ }^{15,42}$, and the few surviving pups are of smaller stature compared to control (Fig. 3a). We aimed at focusing on symN activity and thus chose E14.5, the embryonic stage where the superior cervical ganglia (SCG) formed recently ${ }^{44-48}$ and their size is not different between control and FD yet. Previous reports have shown that the gross loss of progenitor cell mass in sympathetic ganglia begins before E17. $5^{13}$. Accordingly, we did not observe an embryo body size difference between FD and control (Fig. 3b, C). We dissected the SCG (Sup. Fig. 6b), dissociated the symNs and cultured them for 7 days, before measuring neural activity via MEA (Fig. 3d). The SCG were similar in size (Fig. 3e) and the symNs expressed the appropriate markers (Fig. 3f). SCGs from control and FD mice were plated evenly in MEA dishes (Fig. 3h, top) and mouse elp 1 was indeed knocked-out in FD, as no elp1 protein was detectable in the FD cultures 
(Fig. 3h, bottom). FD and control symNs showed similar neurite development (Fig. 3h). We then compared symN activity from control and CKO mice for one week, showing that FD symNs fire spontaneously at a higher rate compared to WT neurons (Fig. 3i, j), consistent with our findings in the iPSC-based symN model. Finally, we found that SCG neurons start to degenerate from day 7 in vitro, supporting the notion that hyperactivity is detrimental, as seen in other systems ${ }^{17}$ (Sup. Fig. 6c). This data supports the finding that sympathetic neurons are spontaneously hyperactive in FD, both in hPSCderived symNs and as well as in symNs derived from two FD mouse models.

\section{Defects in norepinephrine transporter (NET) underlie sympathetic neuron hyperactivity in Familial Dysautonomia}

We next aimed at a better understanding of the molecular mechanism underlying symN hyperactivity in FD. $99.5 \%$ of all FD patients, including the patient's cells analyzed here ${ }^{4}$, harbor a homozygous mutation in ELP1. To examine whether hyperactivity is the direct consequence of the ELP1 mutation, we performed symN differentiations using the ELP1 rescued line, iPSC-EPL $1_{\text {rescued }}{ }^{-T 6.5}$, in which the ELP1 mutation is heterozygously corrected by CRISPR-cas 9 from the iPSC-FD-S2 line ${ }^{4}$. Surprisingly, MEA recording over time revealed that ELP1-rescued FD symNs are still spontaneously hyperactive (Fig. 4a, b), suggesting a mechanism that is indirectly dependent or independent of ELP1 explaining symN hyperactivity.

We hypothesize that hyperactivity in symNs may be caused by defects in the incoming signal (triggering/regulating action potentials) or at the outgoing signal (neurotransmitter release/re-uptake, (Fig. 4c). To investigate the first question, we compared a selection of receptors and transporters that regulate neuronal electrophysiology and have been reported in symNs, including nicotinic receptors (CHRNs), sodium channels $\left(\mathrm{Na}_{\mathrm{v}}\right)$, potassium channels ( $\mathrm{KCNs}$ and Maxi-K), GABA receptor $(\mathrm{GA} A \mathrm{R})$ and calcium channels (P2RX and $\mathrm{Ca}_{\mathrm{v}}$ ). We assessed differential expression thereof and found no significant difference between control and FD (Fig. 4d). We further blocked the incoming signal with the sodium channel blocker tetrodotoxin (TTX) in both control and FD symNs. We found no change in hyperactivity (Fig. 4e), thus confirming that it is caused by a cell-intrinsic mechanism. This result also reduces the potential concern that autapses, which have been shown to form in ex-vivo cultured symNs ${ }^{49}$, lead to FD hyperactivity, since TTX blocks incoming signals from potential self-innervation/stimulation.

We next assessed outgoing signals that could trigger hyperactivity. Expression of VMAT, important for NE vesicle transport, a2AR and $\beta 2 A R s$, involved with NE re-uptake were not expressed differently. However, $\mathrm{NET}$, which reuptakes nearly $90 \%$ of secreted $\mathrm{NE}$, and thus plays a critical role in extracellular NE clearance was expressed significantly lower in FD symN cell bodies and axons (Fig. 4f). NET deficiency has been reported in multiple neural disorders in which SNS dysregulation is involved ${ }^{50,51}$. We confirmed reduced NET protein in FD symNs by IF (Fig. 4g) and showed reduced uptake of fluorescently labeled, synthetic NE by NET in FD symNs, using a NET reuptake assay (Fig. 4h, i). Less NETs on the surface of FD symNs is expected to lead to reduced NE levels inside the cell and excessive NE levels in the extracellular space in FD. Indeed, we showed diminished (though not significantly) NE levels inside FD 
symNs (Fig. 4j), using a novel NE tracker NS510, which is a turn-on probe that allows live cell imaging of intracellular NE dynamics ${ }^{52}$. Accordingly, ELISA measurements of $\mathrm{NE}$ in symN media revealed that in FD symNs more NE is present in the extracellular space (Fig. 4k). For the NET protein to be trafficked to the cell surface and thus become functional, it has to be phosphorylated and glycosylated ${ }^{53}$. Thus, we assessed those modifications on NET in FD and control symNs. We did not detect a significant difference in glycosylation (Fig. 4l), indicating that NET function may not be affected beyond the lower expression levels.

Finally, to test if NET inhibition is sufficient to induce the hyperactive phenotype, we treated healthy control symNs with nomifensine, a norepinephrine-dopamine reuptake inhibitor (NETi) for 24-72 hours to allow the accumulation of extracellular NE and recorded neural activities by MEA. In this analysis, control symNs gradually become hyperactive (Fig. $4 \mathrm{~m}$ ), indicating that indeed NET deficiency in FD symNs may underlie intrinsic hyperactivity. In sum, these results indicate that symNs in FD are intrinsically hyperactive due to defects in auto-regulation of their neurotransmitter (Fig. 4n), which leads to increased activation of their target tissue.

\section{Mini drug screen reveals potential treatments of hyperactive FD symNs}

With these exciting findings, we wanted to test whether this hPSC model system could be used as a platform to test drugs and therefore as a future drug discovery tool. To do so, we selected several drugs whose therapeutic potential have been studied at the clinical or experimental level in FD or other PNS diseases. We tested those drugs on day 35 symNs, the timepoint when hyperactivity in FD symNs first peaks, yet neurodegeneration has not occurred. Dexmedetomidine (Dexmed) is a novel selective a2-AR enhancer, shown to relieve dysautonomic crisis symptoms in some FD patients ${ }^{54}$. Carbidopa is a selective AAAD inhibitor that blocks NE and DA synthesis; its effect of rescuing failed baroreflex functions were shown in some FD patients ${ }^{55}$. We found that indeed, both drugs reduce the exaggerated firing activity of FD symNs in our model (Fig. 5a, b and Sup. Fig. 7a), supporting the notion that our hPSCbased model is useful for drug testing. We next sought to treat FD symNs by targeting the NET pathway. Clozapine is an atypical antipsychotic medicine, which has been demonstrated to elevate NET expression in chromaffin cells ${ }^{56}$. Again, clozapine reduces FD symN hyperactivity significantly in our model (Fig. $5 \mathrm{c}$ and Sup. Fig. 7b). Lastly, propranolol, a $\beta 2 A R$ inhibitor reduces FD hyperactivity as well (Fig. 5d), in fact when both control and FD neurons are treated with propranolol, FD's firing is significantly more reduced (Fig. 5e). Together, these results highlight the usefulness of our modeling platform to assess drugs for FD patients and together with our NETi result on control symNs (Fig. 4m), we show that manipulating NET function is an effective strategy for modulating symN activity.

Next, we aimed to test drugs that have been used in PNS-related neural disorders, but not in FD to our knowledge. Previous research in ALS, a neurodegenerative disease with motor neuron denervation, revealed motor neuron hyperactivity due to potassium channel malfunction and identified flupiritine as a modulator ${ }^{17}$. We tested this drug on FD symNs, and indeed found that flupiritine decreased FD 
hyperactivity (Fig. 5f). Finally, we examined NSC87877 and BGP-15, two drugs that have been studied to prevent PNS neuron neurodegeneration ${ }^{14,16}$. NSC87877 is a tyrosine phosphatase inhibitor shown to prevent FD symN death from impaired retrograde signaling in FD mice ${ }^{16}$. BGP-15 is a small hydroxylamine compound that improves mitochondrial function ${ }^{14}$. We found that treatment of FD symNs with NSC87877 or BGP-15 does not rescue hyperactivity (Fig. 5g, h and Sup. Fig. 7c, d), likely due to their mechanism of action that targets neuronal survival rather than activity. All drugs used in this study do not affect symN survival (Fig. 5i). Finally, we compared the responses of control and FD symNs after propranolol treatment at the same dosage and found that propranolol inhibits FD symN activity even more compared to its action in control neurons (Fig. 5e). This may imply that $\beta_{2} A R$ signaling in FD symns is hypersensitized, further driving the loop of hyperactivity.

Our symN platform and FD model are instrumental for testing current drugs and thus promising for future drug discovery attempts. Furthermore, the results from these drug treatments further strengthen our conclusion about the molecular mechanism underlying symN hyperactivity. In healthy symNs (Fig. 6, top) $\mathrm{NE}$ is released into the extracellular space and about $10 \%$ of it is bound by the target tissue. Another $10 \%$ is taken up by $a_{2} A R$ on the neuron itself, which signals to inhibit further NE release and $10 \%$ is taken up by the $\beta_{2} A R$, which signals to activate NE release. The final $70 \%$ of NE is taken up via NET. FD symNs have less NET molecules available (Fig. 6, bottom), thus NE is depleted inside the cells and in excess outside the cells. That excess can bind to both $a_{2}-A R$ and $\beta_{2} A R$.

Together, we show that our hPSC-derived symN platform is a powerful tool to select potential drug compounds for future treatment options of SNS hyperactivity in FD.

\section{Discussion}

Few in vitro differentiation protocols have been published to date for the generation of postganglionic symNs from hPSCs ${ }^{24-27}$. Our protocol has the following advantages, which makes it ideal for our FD disease modeling application: 1 . It is highly efficient and produces a relatively pure symN population, i.e. $>90 \% \mathrm{NCCs}^{23}$ and $>75 \%$ neurons, of which $>90 \%$ are peripheral neurons (Sup. Fig. 2). 2. It generates functional and mature symNs and is practical, as we have developed a cryopreservation option (Sup.

Fig. 1i, j). 3. Our protocol recapitulates the proper developmental steps of symN development and was the first protocol to be successfully employed for disease modeling in Zeltner et al. ${ }^{4}$, and here.

FD is a developmental disorder with symptom onset at birth ${ }^{5}$. Accordingly, we show reduced generation of NCCs from FD iPSCs (Fig. 1b-e) and reduced cell numbers and lower cell survival at the sympathoblast stage (Fig. 1f-h). However, the symNs that passed through early development, do mature comparably to control neurons (Fig. 1i-k). These observations correlate with FD patient autopsies that showed reduced volume in the sympathetic ganglia ${ }^{12}$. FD animal data from conditional knock-out (CKO) and other mouse models ${ }^{15}$ are slightly different, in that NCC development ensues normally ${ }^{13,32}$, but neuron numbers are decreased due to maldevelopment ${ }^{13,32}$, defective innervation ${ }^{16,42}$, reduced neuron survival ${ }^{13,16}$ and 
abnormal NGF retrograde transport ${ }^{16,57,58}$. However, the end-result of overall reduced symNs is consistent with FD mouse models.

Using our in vitro disease model for FD, we found that FD symNs are intrinsically hyperactive. We confirmed this finding via electrophysiology, associated gene expression, $\mathrm{Ca}^{2+}$-imaging and in co-cultures with target tissue, where cardiomyocytes beat faster when coupled with FD symNs (Fig. 2). We showed that symN hyperactivity is conserved in two CKO FD (elp $\left.7^{\text {LoxP/LoxP }}\right)$ mouse models, driven by either WNT1-cre or SOX10-cre (Fig. 3, Sup. Fig. 6). Neural hyperactivity has been shown in a variety of neurological disorders and is associated with neurodegeneration. Clifford Woolf's group has reported the causality of motor neuron hyperactivity and neurodegeneration in ALS using patient-iPSC model ${ }^{17}$. It has been suggested that network activity may lead to neurodegeneration in the brain of Alzheimer's disease $(A D)^{59,60}$, and an hPSC-based AD model that recapitulates neural hyperactivity was proposed recently by Stuart Lipton's group ${ }^{18}$. We indeed found that symNs from FD animals, that are hyperactive, show signs of degeneration quickly in culture even in the presence of NGF $(60 \mathrm{ng} / \mathrm{ml})($ Sup. Fig. 6c). Future investigations will show if pharmacological prevention of hyperactivity may prevent neurodegeneration in FD.

The SNS is an important component of the stress response system and is crucial for regulation of body homeostasis. FD patients have difficulties regulating arousal, and stress can trigger dysautnomic crisis ${ }^{6}$. Could it be possible then that in FD symNs are more susceptible to stress signaling from the brain? During a stressful state, the extrahypothalamic areas and hypothalamus in the brain release corticotropin releasing factor (CRF), a stress-related neuropeptide, which has been found to regulate the SNS as well as symN activity ${ }^{35}$. We did find elevated expression of CRF receptors (CRHR1/2) in FD symNs. NPY is another important neuropeptide involved in stress modulation both in the CNS and PNS. In PNS, NPY is mainly produced by symNs, its levels have been positively correlated to symN activity, and NPY released by symNs has a vasoconstriction effect ${ }^{34,61}$. Elevated plasma NPY levels are also found in stress-related disorders, such as hypertension and heart failure ${ }^{34}$. Indeed, we detected elevated NPY levels in FD symNs, supporting the hyperactivity phenotype in FD symNs, but also suggesting that FD SNS may be more vulnerable to stress stimulation.

We hypothesize that intrinsic defects within FD symNs are the drivers that lead to hyperactivity, which might further trigger other FD phenotypes and symptoms, including dysautonomic crisis and neurodegeneration. Literature from other SNS related disorders supports this notion. For instance, symN neurotransmitter switching from NE to epinephrine in prehypertensive rats ${ }^{62}$ indicates that symN abnormalities prior to the onset of cardiovascular symptoms may sensitize the SNS and make it more vulnerable to disease-related stimuli. Another study showed that hypertensive symNs were able to change the metabolism of healthy cardiomyocytes to induce a hypertensive state. Vice versa, when hypertensive cardiomyocytes were co-cultured with healthy symNs, the defective response was suppressed ${ }^{63}$. 
Thus, we looked deeper into the molecular mechanism underlying FD symN hyperactivity. We showed that NET expression is reduced in FD, which leads to an accumulation of NE in the extracellular space and to diminished NE inside the cells compared to control (Fig. 4). NET deficiency has been linked to several SNS-related cardiovascular syndromes, such as orthostatic intolerance (OI), postural tachycardia syndrome (POTS) ${ }^{50,64}$, and stress-induced cardiomyopathy ${ }^{51}$, both in patients and animal models ${ }^{51,65}$. Furthermore, a study in patients with hypertension revealed that NE reuptake function was impaired, while the baroreflex control remained unaffected ${ }^{66}$. We also show that NET inhibition is sufficient to trigger symN hyperactivity in healthy hPSCs (Fig. 4m). We further found that NET protein in FD is functional, i.e. glycosylation is not affected (Fig. 4l), thus the issue is expression of the protein itself. As a consequence, in FD excessive NE accumulates in the extracellular space. This, extra NE is bound by $a_{2} A R$, which inhibits more NE release and $b_{2} A R$, which further activates more NE release ${ }^{62,67-69}$. Theoretically, these effects might cancel each other. However, we found that the reduction in hyperactivity after treatment with propranolol, $a b_{2} A R$ inhibitor is more pronounced in FD compared to propranolol treated control symNs (Fig. 5e). Thus, in FD the $b_{2} A R$ might be hypersensitized, which might maintain a feed forward loop, which drives hyperactivity in FD symNs. So, in a FD patient, early embryonic hyperactivity of symNs may explain later degeneration of the cells as well as hypersensitization of $b_{2} A R$ receptors, that together might be the important players for dysautonomic crisis. In our in vitro system, it remains to be shown when measurable degeneration signs begin and if they can be pharmacologically countered, which is a future goal of our work. In patients, indirect measurements of symN activity at rest, i.e. not in crisis, have not shown symN hyperactivity ${ }^{31}$. This discrepancy might have several explanations: (i) Such measurements are indirect, including skin conductance or heart rate assessments ${ }^{21}$ as it is not feasible to directly measure symNs in a patient. Thus, at rest symN hyperactivity might not be strong enough to translate to the downstream target. (ii) In our in vitro, miniature model system, symNs are isolated and thus the released NE is bathing the entire neuron, which provides more opportunity to activate its firing. In comparison, in vivo the excess NE would mainly affect the distal axonal region and thus require more activation, such as during stress-triggered crisis to start hyperactivity in a crisis. (iii) In vivo, in a whole organism, the counteracting parasympathetic nervous system is operating as well.

$99.5 \%$ of all FD patients, including the FD-iPSCs assessed here carry the identical ELP1 mutation ${ }^{31}$. It has been shown that elp1 deficiency preferentially reduces translation of long and AA-rich mRNAs ${ }^{3}$ and increases translation of small AG-biased mRNAs. In a proteomic analysis of the dorsal root ganglia from the Wnt1-cre;Elp1 CKO mouse used here, due to the loss of elongator function, several thousand proteins were expressed at altered levels. Altered pathways included fundamental pathways such as exocytosis, protein transport and chromatin silencing and included changes to histones and transcription factors 3,70 . Our results however also showed that genetic rescue of the FD ELP1 mutation does not reverse hyperactivity (Fig. 4a, b), nor the reduction of NET in symNs (Fig. 4g). One explanation for that observation could be the downstream consequence of altered developmentally-timed genetic programs due to the loss of Elp1 expression and hence they cannot be re-set once the nervous system is mature. Another explanation might be that an additional mechanism is at play. We have shown previously that a 
modifier mutation in $\angle A M B 4$ is present in the patients from whom the here assessed iPSCs are derived. Thus, while at the moment unclear how, it is possible that the interplay of ELP1, NET and LAMB4 might lead to the maintenance of symN hyperactivity in the ELP1-rescued line $\mathrm{P}^{71,72}$.

Finally, we aimed at testing if our symN disease modeling platform is useful for drug screening and drug testing. We found that in this platform, drugs currently given to FD patients for dysautonomic crisis, i.e. dexmedetomidine ${ }^{54}$ and carbidopa ${ }^{55}$ were able to reduce hyperactivity. Additionally, clozapine and flupiritine also could reduce hyperactivity. In contrast, NSC87877 and BGP-15 do not affect FD symN activity in our model. This might be due to the short-term treatment used here and the fact that these drugs have been shown to prevent neurodegeneration. In the future, we aim to establish neurodegeneration in our system and to test if these drugs may prevent it here as well. Together, our drug testing results support our model of the mechanism of defective NE trafficking and NET reuptake, through which hyperactivity in FD is generated and maintained (see model in Sup. Fig. 8). These results also indicate that this platform will be useful for drug screening approaches to identify and test novel compounds that could treat FD or other SNS-related disorders.

\section{Declarations}

Acknowledgements. We thank Dr. Timothy Glass and his group for providing us with the NS510 probe, and Dr. Fikiri Avci and his group for access to equipment for fluorescence ELISA measurements. We also thank Dr. James Lauderdale, Dr. Stephen Dalton, Dr. Jesse Shank, Dr. Chia Wei Huang and Zhonghou Wang for critical discussions and technical support. This work was funded by faculty start-up funds from the University of Georgia to N.Z. and NIH/NINDS 1R01NS114567-01A1 to N.Z., NIH/NIDCD R21DC018089 to HX.L., and R01 DK117473 to F.L..

Author contributions. HFW conceived, conducted and analyzed experiments, wrote on manuscript. WY and $\mathrm{JC}$ conducted animal tissue processing. $\mathrm{FL}$ and $\mathrm{HXL}$ provided support, advice and mice for animal experiments. NZ conceived, designed and lead the study, provided financial and administrative support, edited and approved the manuscript.

Competing interests. The authors have no competing interests.

\section{References}

1. Slaugenhaupt, S.A., et al. Tissue-specific expression of a splicing mutation in the IKBKAP gene causes familial dysautonomia. Am J Hum Genet 68, 598-605 (2001).

2. Kojic, M. \& Wainwright, B. The Many Faces of Elongator in Neurodevelopment and Disease. Front Mol Neurosci 9, 115 (2016).

3. Goffena, J., et al. Elongator and codon bias regulate protein levels in mammalian peripheral neurons. Nat Commun 9, 889 (2018). 
4. Zeltner, N., et al. Capturing the biology of disease severity in a PSC-based model of familial dysautonomia. Nat Med 22, 1421-1427 (2016).

5. Norcliffe-Kaufmann, L., Slaugenhaupt, S.A. \& Kaufmann, H. Familial dysautonomia: History, genotype, phenotype and translational research. Prog Neurobio/ 152, 131-148 (2017).

6. Norcliffe-Kaufmann, L., Palma, J.A. \& Kaufmann, H. Mother-induced hypertension in familial dysautonomia. Clin Auton Res 26, 79-81 (2016).

7. Axelrod, F.B., Zupanc, M., Hilz, M.J. \& Kramer, E.L. Ictal SPECT during autonomic crisis in familial dysautonomia. Neurology 55, 122-125 (2000).

8. Axelrod, F.B., Goldberg, J.D., Ye, X.Y. \& Maayan, C. Survival in familial dysautonomia: Impact of early intervention. J Pediatr 141, 518-523 (2002).

9. Clayson, D., Welton, W. \& Axelrod, F.B. Personality development and familial dysautonomia. Pediatrics 65, 269-274 (1980).

10. Norcliffe-Kaufmann, L., Axelrod, F. \& Kaufmann, H. Afferent baroreflex failure in familial dysautonomia. Neurology 75, 1904-1911 (2010).

11. Norcliffe-Kaufmann, L.J., Axelrod, F.B. \& Kaufmann, H. Cyclic vomiting associated with excessive dopamine in Riley-day syndrome. J Clin Gastroenterol 47, 136-138 (2013).

12. Pearson, J. \& Pytel, B.A. Quantitative studies of sympathetic ganglia and spinal cord intermediolateral gray columns in familial dysautonomia. J Neurol Sci 39, 47-59 (1978).

13. George, L., et al. Familial dysautonomia model reveals Ikbkap deletion causes apoptosis of Pax3+ progenitors and peripheral neurons. Proc Natl Acad Sci U S A 110, 18698-18703 (2013).

14. Ohlen, S.B., Russell, M.L., Brownstein, M.J. \& Lefcort, F. BGP-15 prevents the death of neurons in a mouse model of familial dysautonomia. Proc Natl Acad Sci U S A 114, 5035-5040 (2017).

15. Lefcort, F., Mergy, M., Ohlen, S.B., Ueki, Y. \& George, L. Animal and cellular models of familial dysautonomia. Clin Auton Res 27, 235-243 (2017).

16. Li, L., Gruner, K. \& Tourtellotte, W.G. Retrograde nerve growth factor signaling abnormalities in familial dysautonomia. J Clin Invest 130, 2478-2487 (2020).

17. Wainger, B.J., et al. Intrinsic membrane hyperexcitability of amyotrophic lateral sclerosis patientderived motor neurons. Cell Rep 7, 1-11 (2014).

18. Ghatak, S., et al. Mechanisms of hyperexcitability in Alzheimer's disease hiPSC-derived neurons and cerebral organoids vs isogenic controls. Elife 8(2019). 
19. Li, D., et al. Abnormal intracellular calcium homeostasis in sympathetic neurons from young prehypertensive rats. Hypertension 59, 642-649 (2012).

20. Ohno, T., Shimizu, T., Kato, S., Hayashi, H. \& Hirai, S. Effect of tamsulosin hydrochloride on sympathetic hyperactivity in amyotrophic lateral sclerosis. Auton Neurosci 88, $94-98$ (2001).

21. Zygmunt, A. \& Stanczyk, J. Methods of evaluation of autonomic nervous system function. Arch Med Sci 6, 11-18 (2010).

22. Saito-Diaz, K. \& Zeltner, N. Induced pluripotent stem cells for disease modeling, cell therapy and drug discovery in genetic autonomic disorders: a review. Clin Auton Res 29, 367-384 (2019).

23. Wu, H.F. \& Zeltner, N. Efficient Differentiation of Postganglionic Sympathetic Neurons using Human Pluripotent Stem Cells under Feeder-free and Chemically Defined Culture Conditions. J Vis Exp (2020).

24. Frith, T.J.R. \& Tsakiridis, A. Efficient Generation of Trunk Neural Crest and Sympathetic Neurons from Human Pluripotent Stem Cells Via a Neuromesodermal Axial Progenitor Intermediate. Curr Protoc Stem Cell Biol 49, e81 (2019).

25. Kirino, K., Nakahata, T., Taguchi, T. \& Saito, M.K. Efficient derivation of sympathetic neurons from human pluripotent stem cells with a defined condition. Sci Rep 8, 12865 (2018).

26. Oh, Y., et al. Functional Coupling with Cardiac Muscle Promotes Maturation of hPSC-Derived Sympathetic Neurons. Cell Stem Cell 19, 95-106 (2016).

27. Saito-Diaz, K., Wu, H.F. \& Zeltner, N. Autonomic Neurons with Sympathetic Character Derived From Human Pluripotent Stem Cells. Curr Protoc Stem Cell Bio/ 49, e78 (2019).

28. Katzenell, S., Cabrera, J.R., North, B.J. \& Leib, D.A. Isolation, Purification, and Culture of Primary Murine Sensory Neurons. Methods Mol Biol 1656, 229-251 (2017).

29. Misumi, S., et al. Enhanced neurogenesis from neural progenitor cells with G1/S-phase cell cycle arrest is mediated by transforming growth factor beta1. Eur J Neurosci 28, 1049-1059 (2008).

30. Fattahi, F., et al. Deriving human ENS lineages for cell therapy and drug discovery in Hirschsprung disease. Nature 531, 105-109 (2016).

31. Dietrich, P. \& Dragatsis, I. Familial Dysautonomia: Mechanisms and Models. Genet Mol Bio/39, 497-514 (2016).

32. Hunnicutt, B.J., Chaverra, M., George, L. \& Lefcort, F. IKAP/Elp1 is required in vivo for neurogenesis and neuronal survival, but not for neural crest migration. PLoS One 7, e32050 (2012).

33. Usui, D., et al. Selective activation of the sympathetic ganglia by centrally administered corticotropin-releasing factor in rats. Auton Neurosci 146, 111-114 (2009). 
34. Tan, C.M.J., et al. The Role of Neuropeptide Y in Cardiovascular Health and Disease. Front Physiol 9, 1281 (2018).

35. Deussing, J.M. \& Chen, A. The Corticotropin-Releasing Factor Family: Physiology of the Stress Response. Physiol Rev 98, 2225-2286 (2018).

36. McDonough, S.I., Cseresnyes, Z. \& Schneider, M.F. Origin sites of calcium release and calcium oscillations in frog sympathetic neurons. J Neurosci 20, 9059-9070 (2000).

37. Padmanabhan Nair, V., et al. Activation of HERV-K(HML-2) disrupts cortical patterning and neuronal differentiation by increasing NTRK3. Cell Stem Cell (2021).

38. Cederquist, G.Y., et al. A Multiplex Human Pluripotent Stem Cell Platform Defines Molecular and Functional Subclasses of Autism-Related Genes. Cell Stem Cell 27, 35-49 e36 (2020).

39. Lin, Y., et al. Heparin Promotes Cardiac Differentiation of Human Pluripotent Stem Cells in Chemically Defined Albumin-Free Medium, Enabling Consistent Manufacture of Cardiomyocytes. Stem Cells Transl Med 6, 527-538 (2017).

40. Lin, Y. \& Zou, J. Differentiation of Cardiomyocytes from Human Pluripotent Stem Cells in Fully Chemically Defined Conditions. STAR Protoc 1(2020).

41. Axelrod, F.B., Glickstein, J.S., Weider, J., Gluck, M.C. \& Friedman, D. The effects of postural change and exercise on renal haemodynamics in familial dysautonomia. Clin Auton Res 3, 195-200 (1993).

42. Jackson, M.Z., Gruner, K.A., Qin, C. \& Tourtellotte, W.G. A neuron autonomous role for the familial dysautonomia gene ELP1 in sympathetic and sensory target tissue innervation. Development 141, 24522461 (2014).

43. Yu, W., et al. Taste buds are not derived from neural crest in mouse, chicken, and zebrafish. Dev Biol 471, 76-88 (2021).

44. Armstrong, A., Ryu, Y.K., Chieco, D. \& Kuruvilla, R. Frizzled3 is required for neurogenesis and target innervation during sympathetic nervous system development. J Neurosci 31, 2371-2381 (2011).

45. Fagan, A.M., et al. TrkA, but not TrkC, receptors are essential for survival of sympathetic neurons in vivo. J Neurosci 16, 6208-6218 (1996).

46. Hendry, I.A. Cell division in the developing sympathetic nervous system. J Neurocyto/ 6, 299-309 (1977).

47. Rothman, T.P., Gershon, M.D. \& Holtzer, H. The relationship of cell division to the acquisition of adrenergic characteristics by developing sympathetic ganglion cell precursors. Dev Bio/ 65, 322-341 (1978). 
48. Rubin, E. Development of the rat superior cervical ganglion: ganglion cell maturation. $J$ Neurosci $\mathbf{5}$, 673-684 (1985).

49. Ma, H. \& Mochida, S. A cholinergic model synapse to elucidate protein function at presynaptic terminals. Neurosci Res 57, 491-498 (2007).

50. Robertson, D., et al. Familial orthostatic tachycardia due to norepinephrine transporter deficiency. Ann N Y Acad Sci 940, 527-543 (2001).

51. Schroeder, C. \& Jordan, J. Norepinephrine transporter function and human cardiovascular disease. Am J Physiol Heart Circ Physio/ 303, H1273-1282 (2012).

52. Zhang, L., Liu, X.A., Gillis, K.D. \& Glass, T.E. A High-Affinity Fluorescent Sensor for Catecholamine: Application to Monitoring Norepinephrine Exocytosis. Angew Chem Int Ed Eng/ 58, 7611-7614 (2019).

53. Khan, A.W., Corcoran, S.J., Esler, M. \& El-Osta, A. Epigenomic changes associated with impaired norepinephrine transporter function in postural tachycardia syndrome. Neurosci Biobehav Rev 74, 342355 (2017).

54. Dillon, R.C., et al. Dexmedetomidine for refractory adrenergic crisis in familial dysautonomia. Clin Auton Res 27, 7-15 (2017).

55. Norcliffe-Kaufmann, L., Palma, J.A., Martinez, J. \& Kaufmann, H. Carbidopa for Afferent Baroreflex Failure in Familial Dysautonomia: A Double-Blind Randomized Crossover Clinical Trial. Hypertension 76, 724-731 (2020).

56. Mandela, P. \& Ordway, G.A. The norepinephrine transporter and its regulation. J Neurochem 97, 310-333 (2006).

57. Naftelberg, S., et al. Phosphatidylserine Ameliorates Neurodegenerative Symptoms and Enhances Axonal Transport in a Mouse Model of Familial Dysautonomia. PLoS Genet 12, e1006486 (2016).

58. Ghosh, S., Li, L. \& Tourtellotte, W.G. Retrograde nerve growth factor signaling abnormalities and the pathogenesis of familial dysautonomia. Neural Regen Res 16, 1795-1796 (2021).

59. Crossley, N.A., et al. The hubs of the human connectome are generally implicated in the anatomy of brain disorders. Brain 137, 2382-2395 (2014).

60. de Haan, W., Mott, K., van Straaten, E.C., Scheltens, P. \& Stam, C.J. Activity dependent degeneration explains hub vulnerability in Alzheimer's disease. PLoS Comput Biol 8, e1002582 (2012).

61. Kataoka, Y., et al. The involvement of neuropeptide $\mathrm{Y}$ in the antimuricide action of noradrenaline injected into the medial amygdala of olfactory bulbectomized rats. Pharmacol Biochem Behav 28, 101103 (1987). 
62. Bardsley, E.N., Davis, H., Buckler, K.J. \& Paterson, D.J. Neurotransmitter Switching Coupled to betaAdrenergic Signaling in Sympathetic Neurons in Prehypertensive States. Hypertension 71, 1226-1238 (2018).

63. Larsen, H.E., Lefkimmiatis, K. \& Paterson, D.J. Sympathetic neurons are a powerful driver of myocyte function in cardiovascular disease. Sci Rep 6, 38898 (2016).

64. Shannon, J.R., et al. Orthostatic intolerance and tachycardia associated with norepinephrinetransporter deficiency. N Engl J Med 342, 541-549 (2000).

65. Keller, N.R., et al. Norepinephrine transporter-deficient mice exhibit excessive tachycardia and elevated blood pressure with wakefulness and activity. Circulation 110, 1191-1196 (2004).

66. Schlaich, M.P., et al. Sympathetic augmentation in hypertension: role of nerve firing, norepinephrine reuptake, and Angiotensin neuromodulation. Hypertension 43, 169-175 (2004).

67. De Blasi, A. Beta-adrenergic receptors: structure, function and regulation. Drugs Exp Clin Res 16, 107-112 (1990).

68. Graham, R.M. Adrenergic receptors: structure and function. Cleve Clin J Med 57, 481-491 (1990).

69. Hein, L., Altman, J.D. \& Kobilka, B.K. Two functionally distinct alpha2-adrenergic receptors regulate sympathetic neurotransmission. Nature 402, 181-184 (1999).

70. Cameron, B., et al. Loss of Elp1 perturbs histone H2A.Z and the Notch signaling pathway. Biol Open 10(2021).

71. Ferrer-Ferrer, M. \& Dityatev, A. Shaping Synapses by the Neural Extracellular Matrix. Front Neuroanat 12, 40 (2018).

72. Lam, D., et al. Tissue-specific extracellular matrix accelerates the formation of neural networks and communities in a neuron-glia co-culture on a multi-electrode array. Sci Rep 9, 4159 (2019).

73. Lafaille, F.G., et al. Human SNORA31 variations impair cortical neuron-intrinsic immunity to HSV-1 and underlie herpes simplex encephalitis. Nat Med 25, 1873-1884 (2019).

74. Ch'ng, T.H., Flood, E.A. \& Enquist, L.W. Culturing primary and transformed neuronal cells for studying pseudorabies virus infection. Methods Mol Biol 292, 299-316 (2005).

\section{Online Methods}

Stem cell cultures 
All stem cell lines used are listed in Zeltner, $2016^{4}$. Cells were maintained in feeder-free and chemically defined Essential 8 medium (Gibco, A15170-01) on 5 g $/ \mathrm{ml}$ vitronectin coated (Thermo Fisher/Life Technologies, A14700) cell culture plates, according to the manufacturer's instructions.

In vitro differentiations

Sympathetic neuron differentiation. The differentiation protocol is modified from our previous publications ${ }^{4,23}$. hPSCs on day 0 were dissociated by EDTA. After dissociation, cells were mixed in day 01 medium that contains Essential 6 medium (Gibco, A15165-01), 0.4 ng/ml BMP4 (PeproTech, 314-BP), $10 \mu \mathrm{M}$ SB431542 (R\&D Systems, 1614) and 300 nM CHIR99021 (R\&D Systems, 4423), and plated on Geltrex (Invitrogen, A1413202) coated cell culture plates at $1.25 \times 10^{5} / \mathrm{cm}^{2}$ density. On day 2, change the culture medium to day 2-10 medium, that contains Essential 6 medium, $10 \mu \mathrm{M} \mathrm{SB} 431542$ and $0.75 \mu \mathrm{M}$ CHIR99021. Medium is changed every other day. On day 10, NCCs were dissociated by accutase (Coring, AT104500) and resuspended on ultra-low attachment plates (Corning, 07200601 and 07200 602) in day 10-14 medium that contains Neurobasal medium (Gibco, 21103-049), B27 (Gibco, 17502-048), LGlutamine (Thermo Fisher/Gibco, 25030-081), 3 MM CHIR99021 and 10 ng/ml FGF2 (R\&D Systems, 233FB/CF). On day 14, sympathetic neuroblast spheroids were spun down and dissociated by accutase. Cells were plated on PO (Sigma, P3655)/LM (R\&D Systems, 3400-010-01)/FN (VWR/Corning, 47743-654) coated plates at $1 \times 10^{5} / \mathrm{cm}^{2}$ density in symN medium that contains Neurobasal medium, B27, LGlutamine, 25 ng/ml GDNF (PeproTech, 450), 25 ng/ml BDNF (R\&D Systems, 248-BD), 25 ng/ml NGF (PeproTech, 450-01), $200 \mu \mathrm{M}$ ascorbic acid (Sigma, A8960), $0.2 \mathrm{mM}$ dbcAMP (Sigma, D0627) and 0.125 $\mu \mathrm{M}$ retinoic acid (Sigma, R2625, add freshly every feeding). Medium is changed every three days until day 20 , and every week after that. To eliminate contaminating cells, 0.5-1 $\mu \mathrm{M}$ aphidicolin (Cayman, 14007) can be added to the symN medium from day 20-30.

Note: BMP4 quality is highly lot dependent. It is highly recommended to perform titration test and decide the best BMP4 concentration for each batch of BMP4.

CNS neuron differentiation. The differentiation protocol is modified from our previous publications ${ }^{38,73}$. hPSCs on day -1 were dissociated by EDTA. After dissociation, cells were mixed in Essential 8 medium, supplied with $10 \mu \mathrm{M}$ Y-27632 (R\&D Systems, 1254) and plated on Matrigel (Corning, 1:20)-coated plates at $2.5 \times 10^{5} / \mathrm{cm}^{2}$ density. On day 0 , medium is switched to day 0-2 medium, that contains Essential 6 medium, $10 \mu \mathrm{M} \mathrm{SB}, 200 \mathrm{nM}$ LDN193189 (Selleck Chemicals, S2618) and $2 \mu \mathrm{M}$ XAV939 (TOCRIS, 3748). On day 3, feed with day 3-5 medium that contains Essential 6 medium, $10 \mu \mathrm{M} \mathrm{SB}$ and 200nM LDN193189. On day 5-9 feed with medium that contains Essential 6 medium and $10 \mu M$ SB. On day 10, medium was changed to day 10-20 medium that contains Neurobasal medium, B27 (1:1000) and GlutaMAX (Gibco, 35050061). On day 20, neural progenitors were dissociated by accutase and resuspended in day 20-30 medium that contains Neurobasal medium, B27 (1:50), GlutaMAX and $40 \mu \mathrm{M}$ DAPT (R\&D Systems, 2634). From day 30, DAPT is removed from the medium and $25 \mathrm{ng} / \mathrm{ml}$ GDNF, 25 $\mathrm{ng} / \mathrm{ml} \mathrm{BDNF}, 200 \mu \mathrm{M}$ ascorbic acid, $0.2 \mathrm{mM}$ dbcAMP is added to the the final medium. 
Cardiomyocyte differentiation. The differentiation protocol is modified from previous publications 39,40 . hPSCs on day -2 were dissociated shortly by EDTA and split at a 1:5 ratio on plates coated with 1:20 diluted Matrigel, in Essential 8 medium supplied with $10 \mu \mathrm{M}$ Y-27632. Prepare CDBM base medium that contains DMEM/F12 (Gibco, 11320033), $64 \mathrm{mg} / \mathrm{L}$ ascorbic acid, $13.6 \mu \mathrm{g} / \mathrm{L}$ sodium selenium (Sigma, S5261), 10 $\mathrm{gg} / \mathrm{ml}$ transferrin (Sigma, T3309) and Chemically Defined Lipid Concentrate (Gibco, 11905031). Cells were fed with Essential 8 medium until day 0. On day 0, cells were fed with day 0 medium that contains CDBM base and $5 \mu \mathrm{M}$ CHIR99021. Cells were fed daily thereafter. Day 1, 5, 6 medium contains CDBM base and $0.6 \mathrm{U} / \mathrm{ml}$ heparin (STEMCELL Technologies, 07980). Day 2, 3, 4 medium contains CDBM base, $0.6 \mathrm{U} / \mathrm{ml}$ heparin and $3 \mu \mathrm{M}$ XAV. On day $7, \mathrm{CMs}$ were dissociated by accutase and resuspended in final CM medium that contains CDBM base and $20 \mu \mathrm{g} / \mathrm{ml}$ insulin (Sigma, I034 ) at $2 \times 10^{5}$ cells $/ \mathrm{cm}^{2}$ density on Matrigel coated plates. For symN and CM co-culture, day $14 \mathrm{symN}$ progenitors were added on top of day $15 \mathrm{CMs}$ at $1 \times 10^{5} \mathrm{cell} / \mathrm{s} / \mathrm{cm}^{2}$ density in symN medium plus CM medium at a 1:1 ratio.

SCG neuron purification and culture

SOX10-cre;Elp1 Loxp/LoxP CKO mice were provided by Dr. Hong-Xiang Liu's lab at University of Georgia and

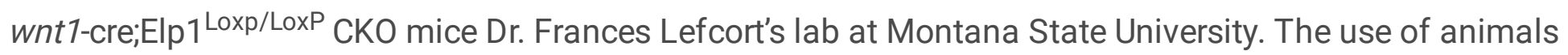
was approved by the Institutional Animal Care and Use Committee at the University of Georgia and Montana State University. The study was performed in compliance with the National Institutes of Health Guidelines for the care and use of animals in research The mouse SCGs at E14.5 were isolated and cultured as previously described ${ }^{13,74}$. In brief, dissected SCGs were washed once by $1 \times$ HBSS and centrifuged at $300 \mathrm{~g}$ for $5 \mathrm{~min}$. SCGs were incubated with $0.25 \mathrm{mg} / \mathrm{ml}$ of trypsin (Thermo Fisher Scientific, 25200056) at $37^{\circ} \mathrm{C}$ for $15 \mathrm{~min}$. After centrifugation, $1 \mathrm{mg} / \mathrm{ml}$ of trypsin inhibitor (Thermo Fisher Scientific, R007100) was added to SCGs and they were incubated for another $5 \mathrm{~min}$ at $37^{\circ} \mathrm{C}$. SCGs were then centrifuged and fully dissociated by pipetting with SCG culture medium that consists of Neurobasal medium, B27, GlutaMAX (according to the manufacturer's instructions) and $60 \mathrm{ng} / \mathrm{ml}$ NGF (5$10 \mu \mathrm{l} / 2$ SCGs). Dissociated SCGs were plated on 96-well BioCircuit or CytoView MEA plate (Axion BioSystems, M768-BIO-96 or M768-tMEA-96W), that were coated with PO/LM/FN, as droplets (5-10 $\mu \mathrm{l} / 2$ SCGs each well) for MEA measurements or on regular cell culture plates (Corning, 3524) for RNA sampling or immunostaining. For MEA plates, droplets were incubated at $37^{\circ} \mathrm{C}$ for $30-45 \mathrm{~min}$, and filled up with $200 \mu \mathrm{l}$ culture medium per well. $10 \mu \mathrm{M}$ Y-27632 can be added to culture medium on the day of plating for improved attachment.

Live cell flow cytometry.

Day 10 NCCs were washed with 1x PBS and dissociated in accutase. The cell/accutase mixture was washed by mixing the solution with FACS buffer that contains 1x DMEM (Life Technologies, 10829-018), $2 \%$ FBS (Atlanta Biologicals, S11150) and $200 \mathrm{mM}$ L-Glutamine. The cells were spun twice at $200 \mathrm{~g}$ for 4 min. Count the cells and incubate $1 \times 10^{6}$ cells with mouse anti-CD49d-PECy7 (Biolegend, 304313, 1:20 in $100 \mu l)$ for 20 min on ice. After incubation, wash cells by mixing the mixture with FACS buffer and 
spinning twice at $200 \mathrm{~g}$ for $4 \mathrm{~min}$. Cells in FACS buffer were kept on ice and analyzed using Beckman Coulter CytoFLEX.

\section{Quantitative RT-qPCR}

Cell lysates were obtained from 1-2 wells of a 24-well plate for each sample and the RNA was extracted using the Trizol reagent (Invitrogen, 15596026). $1 \mu \mathrm{g}$ of total RNA each sample was reverse-transcribed into cDNA using iScript ${ }^{\text {tM }}$ Reverse Transcription Supermix (Bio-Rad, 170884) according to the manufacturer's instruction. RT-qPCR was performed with SYBR green (Bio-Rad) and analyzed with a CFX96 Touch Deep Well Real-Time PCR Detection System (Bio-Rad). The primers used are listed in Sup. Table 1.

\section{Immunofluorescence staining}

Neurons cultured in the desired culture plates were fixed with $4 \%$ paraformaldehyde for 20 min and washed with $1 \times$ PBS. Samples were permeabilized using $0.3 \%$ Triton, $1 \%$ BSA and $3 \%$ goat or donkey serum in $1 \times$ PBS for 20 min. Primary antibodies were added to the cells and incubated at $4{ }^{\circ} \mathrm{C}$ overnight. The next day, cells were washed twice and incubated with secondary antibodies for $1 \mathrm{hr}$ at RT. Images were taken using the Lionheart FX Automated Microscope. The antibodies used here are listed in Sup.

Table 2.

\section{Western blot analysis}

For protein lysate collection, day 35 symNs in one well of 6-well culture plate (Corning) were prepared. After washing with $1 \times$ PBS, cells were lysed by lysis solution that contains 1x RIPA buffer (Sigma-Aldrich, R0278), 1 mM PMSF protease inhibitor (Thermo Fisher Scientific, 36978) and 10x PhosSTOP phosphatase inhibitor (Sigma-Aldrich, 4906845001). Bradford reagent (Bio-Rad, 5000006) was used for measuring protein concentration. For western blotting, $20 \mu \mathrm{g} /$ well of total protein for each sample was loaded and ran in $12 \%$ acrylamide gels. Proteins were then transferred to nitrocellulose membrane and blocked with $5 \%$ skim milk in TBST. The membranes were incubated with primary antibodies including mouse monoclonal anti-hNET (MAb Technologies, NET17-1, 1:1000) and mouse monoclonal anti-c-Fos (Abcam, ab208942, 1:1000) at $4{ }^{\circ} \mathrm{C}$ overnight. Next day, the membranes were washed 3 times with PBST and incubated with goat anti-mouse and goat anti- HRP for $1 \mathrm{hr}$ at RT.

\section{MEA assay.}

SymNs for the extracellular spike measurements were plated on 96-well BioCircuit or CytoView MEA plate, coated with $\mathrm{PO} / \mathrm{LM} / \mathrm{FN}$, at $1 \times 10^{5}$ cells $/ \mathrm{cm}^{2}$ density. If BioCircuit plates were used, it is recommended that additional wells on a regular cell culture plate with visible bottoms (Corning, CLS35) are used at the same condition to monitor the growth of symNs. Neural activity was measured at the desired timepoints using the Maestro Pro multiwell plate reader (Axion BioSystems). 
SymNs for the experiment were plated on 96-well black well/clear bottom plates (Corning, 3603), coated with PO/LM/FN, at $1 \times 10^{5}$ cells $/ \mathrm{cm}^{2}$ density. Day 35 symNs were washed with $1 \times$ HBSS (Gibco) once. For the blank, $200 \mu \mathrm{l}$ of fresh culture medium was added into one well of each experimental groups. For intracellular $\mathrm{Ca}^{2+}$ tracing, $2 \mu \mathrm{M}$ of Fluo-4 AM (TOCRIS, 6255) was made with culture medium and added to duplicated experimental groups $\left(200 \mu \mathrm{l} /\right.$ well). SymNs with Fluo-4 were incubated at $37^{\circ} \mathrm{C}$ for $20 \mathrm{~min}$, followed by a $1 \mathrm{x}$ HBSS wash three times and incubated for 30 minutes with fresh medium. After the incubation, symNs were read using a fluorescence microplate reader (BioTek) immediately at $440 \mathrm{~nm}$ excitation and $520 \mathrm{~nm}$ emission or imaged using the Lionheart FX Automated Microscope.

\section{Norepinephrine imaging}

The NE tracer (NS510) was a kind gift by Timothy Glass's laboratory at University of Missouri, and used according to previous publication ${ }^{52}$. SymNs for the experiment were plated on 96-well black well/clear bottom plates (Corning), coated with PO/LM/FN, at $1 \times 10^{5}$ cells $/ \mathrm{cm}^{2}$ density. Day 35 symNs were washed with $1 x$ HBSS once. For the blank, $200 \mu$ of fresh culture medium was added into one well of each experimental groups. For intracellular NE tracing, $0.5 \mu \mathrm{M}$ of NS510 was made with culture medium and added to duplicated experimental groups $\left(200 \mu \mathrm{l} /\right.$ well). SymNs with NS510 were incubated at $37^{\circ} \mathrm{C}$ for $45 \mathrm{~min}$, followed by a $1 \mathrm{x}$ HBSS wash twice and read using a fluorescence microplate reader (BioTek) immediately at $440 \mathrm{~nm}$ excitation and $520 \mathrm{~nm}$ emission or imaged using a Lionheart FX Automated Microscope.

\section{NET reuptake assay.}

The assay was performed according to manufacturer's instructions (Neurotransmitter Transporter Uptake Assay Kit, Molecular Devices, R6138). SymNs for the experiment were plated on 96-well black well/clear bottom plates (Corning), coated with PO/LM/FN, at $1 \times 10^{5}$ cells $/ \mathrm{cm}^{2}$ density. Day 35 symNs were washed with $1 \mathrm{x}$ HBSS once. For the blank, $200 \mu \mathrm{l} /$ well of $1 \mathrm{xHBSS}$ was added in to one well of each experimental group. For NET reuptake measurement, $100 \mu \mathrm{l}$ of $1 \mathrm{x}$ HBSS with $100 \mu \mathrm{l}$ dye solution/well was added in to 2 wells (duplication is recommended) of each experimental group. The plate was read immediately using a fluorescence microplate reader (BioTek) at $440 \mathrm{~nm}$ excitation and $520 \mathrm{~nm}$ emission.

\section{NE ELISA}

The assay was performed according to manufacturer's instructions (EagleBio, NOU39-K01). Media in two wells of a 24-well of day 35 symNs from each experimental group was changed ( $500 \mu \mathrm{l} /$ well) one day before the experiment. 24 hrs later, media from two wells were harvested and pooled together to concentrate the NE. To avoid NE degradation, sample stabilizer included in the kit was added to each sample. Collected media was spun at $300 \mathrm{~g}$ for $5 \mathrm{~min}$ to remove debris. The samples were ready for NE detection or were stored at $-80^{\circ} \mathrm{C}$ for long-term storage.

\section{MTT assay.}


PrestoBlue Viability Reagent (Invitrogen, A13261) was used for measuring symN viability. SymNs for the experiment were plated on 96 -well cell cultured plates, coated with PO/LM/FN, at $1 \times 10^{5}$ cells $/ \mathrm{cm}^{2}$ density (two wells for each experimental group). Media on day 35 neurons was replaced with 1:10 diluted viability reagent with fresh culture medium ( $200 \mu \mathrm{l}$ per well, duplication is recommended), and incubate at $37^{\circ} \mathrm{C}$ for $3 \mathrm{hrs}$. $200 \mu \mathrm{l}$ of culture medium without dye solution was added to one well of each group for blank measurement. After $3 \mathrm{hrs}, 100 \mu \mathrm{l}$ of medium and dye mixture of each well was transferred to 96well plates with clear flat-bottom (Corning, 3596), and read using a microplate reader (BioTek) immediately at $570 \mathrm{~nm}$ and $600 \mathrm{~nm}$ absorbance.

\section{$\underline{\text { Statistical analysis }}$}

Data was collected from at least three independent experiments (biological replicates), with multiple technical replicates each. Quantification results were presented as mean \pm SEM. Significance calculation between two experimental groups was analyzed by two-tailed Student's t-test. For multiple group comparison, one-way ANOVA is used followed by Tukey's multiple comparison tests, while two-way ANOVA followed by Šídák multiple comparisons was used for comparisons among different timepoints. All analysis is performed using Prism 9.

\section{Online Methods References}

1. Zeltner, N., et al. Capturing the biology of disease severity in a PSC-based model of familial dysautonomia. Nat Med 22, 1421-1427 (2016).

2. Wu, H.F. \& Zeltner, N. Efficient Differentiation of Postganglionic Sympathetic Neurons using Human Pluripotent Stem Cells under Feeder-free and Chemically Defined Culture Conditions. J Vis Exp (2020).

3. Cederquist, G.Y., et al. A Multiplex Human Pluripotent Stem Cell Platform Defines Molecular and Functional Subclasses of Autism-Related Genes. Cell Stem Cell 27, 35-49 e36 (2020).

4. Lafaille, F.G., et al. Human SNORA31 variations impair cortical neuron-intrinsic immunity to HSV-1 and underlie herpes simplex encephalitis. Nat Med 25, 1873-1884 (2019).

5. Lin, Y., et al. Heparin Promotes Cardiac Differentiation of Human Pluripotent Stem Cells in Chemically Defined Albumin-Free Medium, Enabling Consistent Manufacture of Cardiomyocytes. Stem Cells Trans/ Med 6, 527-538 (2017).

6. Lin, Y. \& Zou, J. Differentiation of Cardiomyocytes from Human Pluripotent Stem Cells in Fully Chemically Defined Conditions. STAR Protoc 1(2020).

7. Ch'ng, T.H., Flood, E.A. \& Enquist, L.W. Culturing primary and transformed neuronal cells for studying pseudorabies virus infection. Methods Mol Biol 292, 299-316 (2005). 
8. George, L., et al. Familial dysautonomia model reveals lkbkap deletion causes apoptosis of Pax3+ progenitors and peripheral neurons. Proc Natl Acad Sci U S A 110, 18698-18703 (2013).

9. Zhang, L., Liu, X.A., Gillis, K.D. \& Glass, T.E. A High-Affinity Fluorescent Sensor for Catecholamine: Application to Monitoring Norepinephrine Exocytosis. Angew Chem Int Ed Eng/ 58, 7611-7614 (2019).

\section{Figures}

Fig 1. Developmental phenotypes in the FD sympathtic lineage

a b

FD vs ctrl

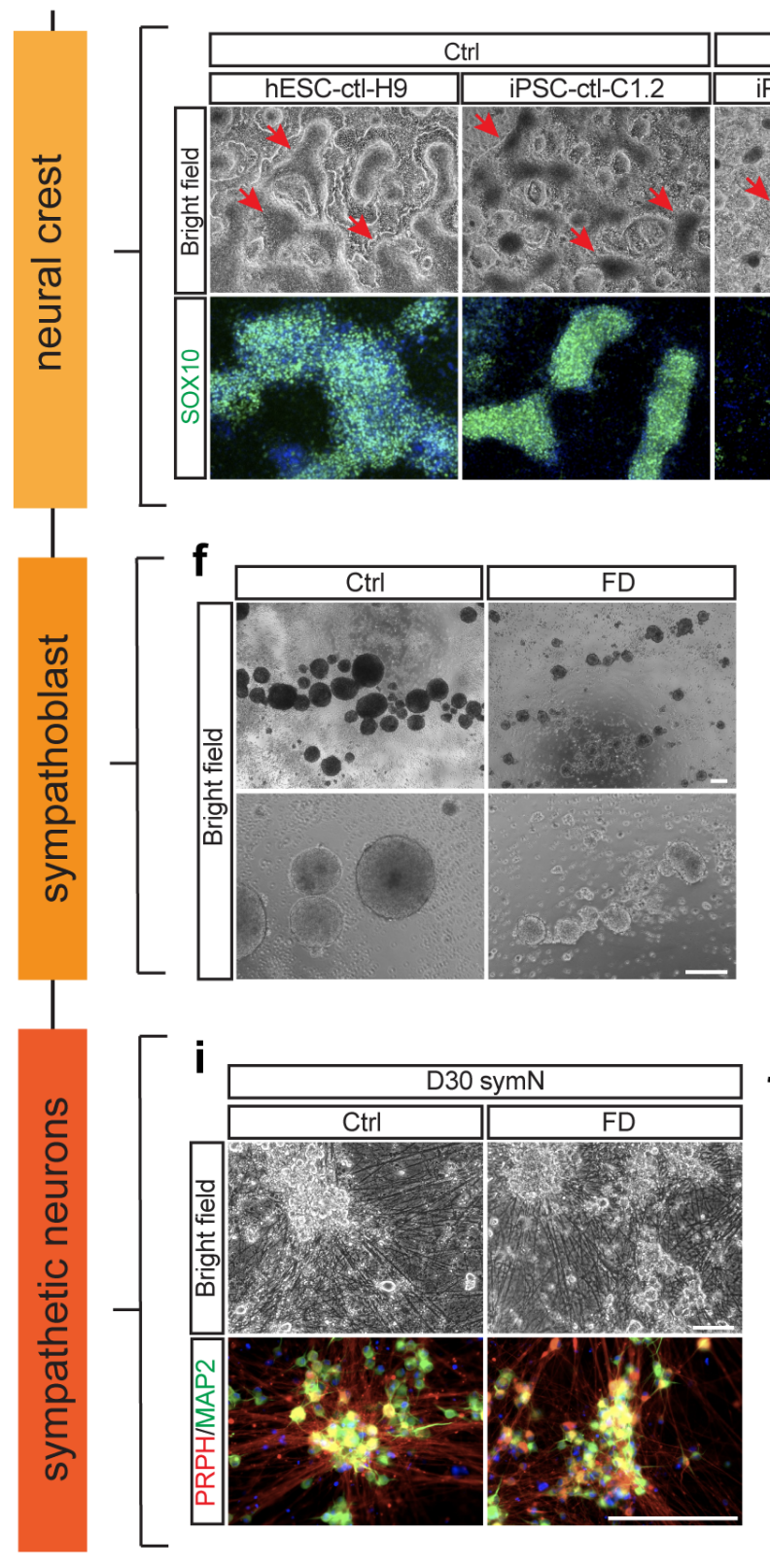

C

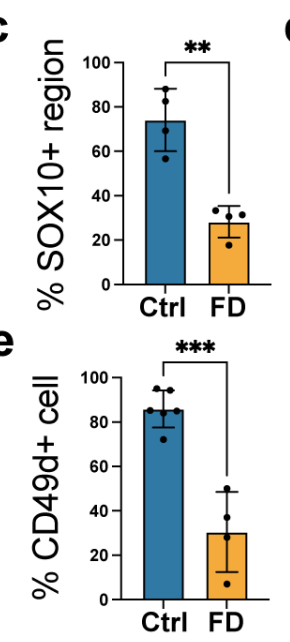

d

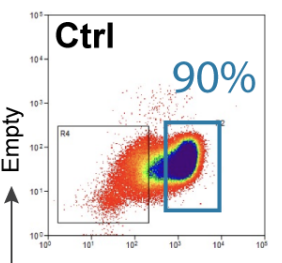

FD

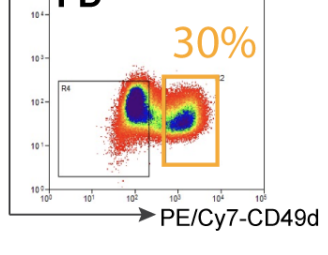

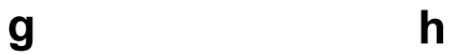
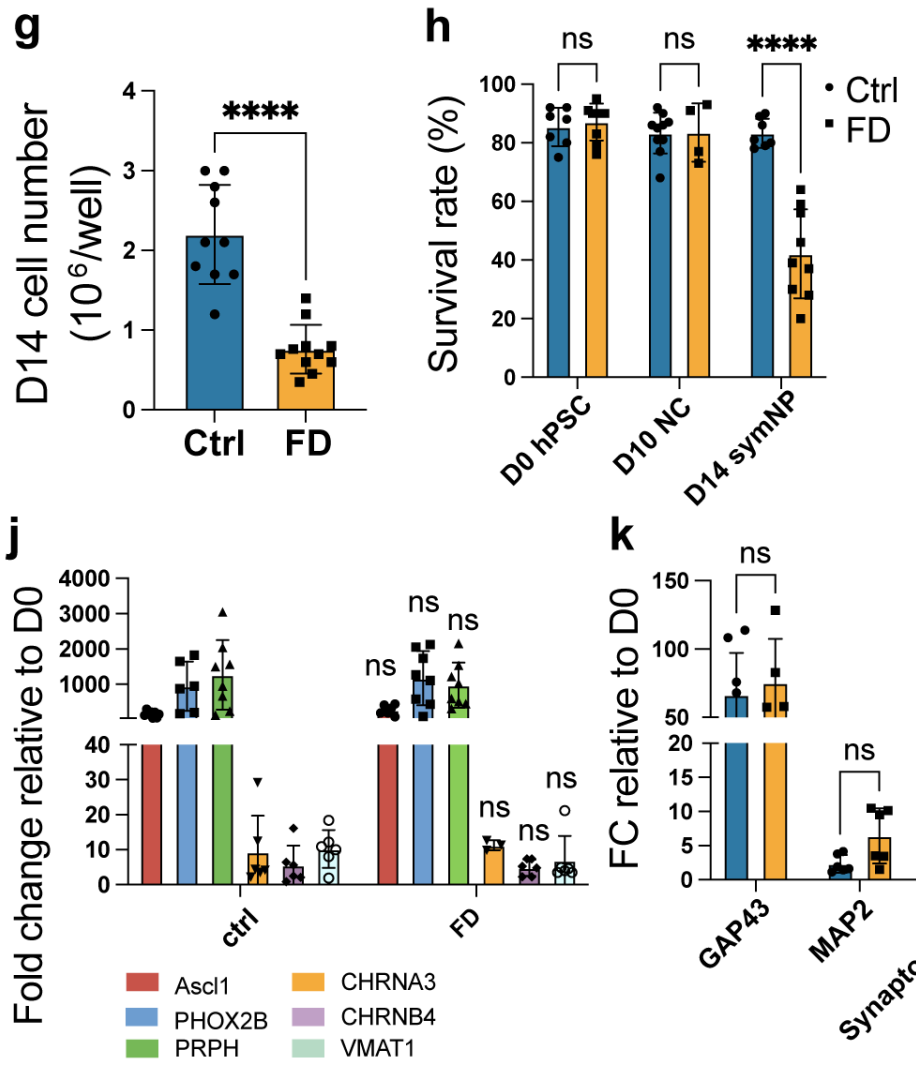

Figure 1 
Developmental phenotypes in FD symNs. (a) Schematic workflow of developmental comparisons during symN differentiation in ctrl and FD groups. (b) Comparison of NCC differentiation on day 10. Red arrows indicate the NC "ridge" structures. Immunostaining in bottom row shows SOX10 (green) positive NCC ridges at high scale. (c) Quantification of SOX10+ cell area in (b). $n=4$ biological replicates. Two-tailed Student's t-test. Error bar represents the SEM. ${ }^{*} p<0.01$. (d) Day 10 NCC number is analyzed by FACS using PE/Cy7-CD49d. Blue rectangle in ctrl and yellow in FD gate CD49d+fractions. (e) Quantification of CD49d+ cell number by FACS. $n=4-6$ biological replicates. Two-tailed Student's t-test. Error bar represents the SEM. ${ }^{* \star *} p<0.001$. (f) Representative sympathoblast spheroids are compared on day14. (g) Day 14 total cell numbers after dissociation is also compared. $n=10-11$ biological replicates. Two-tailed Student's t-test. Error bar represents the SEM. ${ }^{\star \star \star *} p<0.0001$. (h) Survival rates after dissociation are quantified and compared at each stage. SymNP=sympathoblast. $n=4-9$ biological replicates. Multiple unpaired Student's t-test. Error bar represents the SEM. ${ }^{\star \star \star \star} p<0.0001$. (i) Representative differentiated symNs are compared after day 30 in bright field (top row) and PRPH (red)/MAP2 (green) staining (bottom row). (j) RT-qPCR analysis shows similar expressions of symN markers between control and FD. $n=3-8$ biological replicates. Multiple unpaired Student's t-test. Error bar represents the SEM. (k) Gene expression of neurite growth markers are further examined using RT-qPCR and no significant difference is found. $n=4-6$ biological replicates. Multiple unpaired Student's t-test. Error bar represents the SEM. Scale bars represent $200 \mu \mathrm{m}$. In $\mathrm{c}, \mathrm{g}$ and $\mathrm{h}$, data from hESC-ctl-H9 and iPSC-ctl-C1.2 are pooled as control; data from iPSC-FD-S2.3 and iPSC-FD-S3 are pooled as FD. See also Sup. Figs. 1-4. 
Fig 2. FD symNs are hyperactive
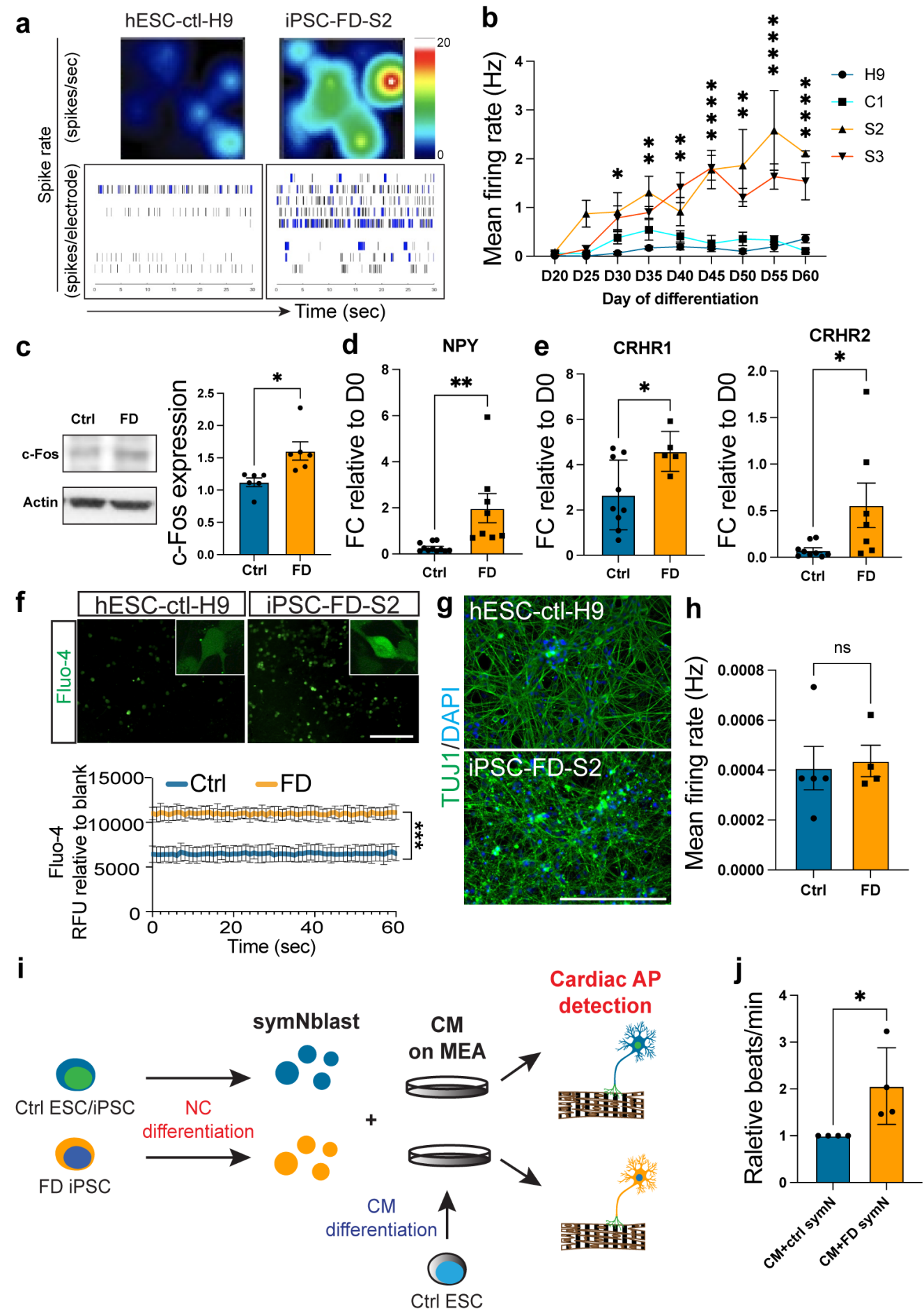

\section{Figure 2}

FD symNs are spontaneously hyperactive. (a) Heatmaps of neural spike rate (top row) and burst maps (bottom row, black bars represent spikes and blue bars represent bursts) on day 35, show representative patterns of FD symN hyperactivity. (b) SymN firing frequencies are compared using MEA in a time course. n=4-14 biological replicates. Two-way ANOVA followed by Šídák multiple comparisons. Control and FD were compared. Error bar represents the SEM. ${ }^{*} P<0.05$, ${ }^{* *} p<0.01,{ }^{* * * *} P<0.0001$. (c) Comparison and 
quantification of c-Fos expression by western blot between control and FD. $n=6$ biological replicates.

Two-tailed Student's t-test. Error bar represents the SEM. *p<0.05. (d-e) RT-qPCR analysis of NPY (d) and CRHR1/2 (e) levels between control and FD. $n=5-10$ biological replicates. Two-tailed Student's t-test. Error bar represents the SEM. ${ }^{*} p<0.05,{ }^{*} \mathrm{P}<0.01$. (f) $\mathrm{Ca} 2+$ image using Fluo-4 shows higher $\mathrm{Ca} 2+$ influx in FD symNs. Images in top row show representative levels of Ca2+ in symNs. ELISA-based time lapse recording in bottom row shows constantly higher Ca2+ levels in FD symNs. $\mathrm{n}=4$ biological replicates. Two-way ANOVA followed by Šídák multiple comparisons. Error bar represents the SEM. ${ }^{* \star \star} p<0.001$. (g-h) CNS neurons differentiated from ctrl and FD hPSCs are Tuj $1+$ (green, g), and show no difference in neural activity via MEA (h). $n=4-5$ biological replicates. Two-tailed Student's t-test. Error bar represents the SEM. (i) Cartoon shows the rationale of symN and cardiomyocyte (CM) co-culture design. Day 14 sympathoblasts from control and FD hPSCs are plated on day 15 cardiomyocytes from hESC-ctl-H9. (j) Quantification of CM beating rate with control or FD symN shows that FD symNs activate CMs at a higher level. $\mathrm{n}=4$ biological replicates. Two-tailed Student's t-test. Error bar represents the SEM. ${ }^{*} \mathrm{P}<0.05$. Scale bars represent $200 \mu \mathrm{m}$. In b, c, d, e, f and j, data from hESC-ctl-H9 and iPSC-ctl-C1.2 are pooled as control; data from iPSC-FD-S2.3 and iPSC-FD-S3 are pooled as FD. See also Sup. Fig. 5 
Fig 3. Mouse SCG hyperactivity
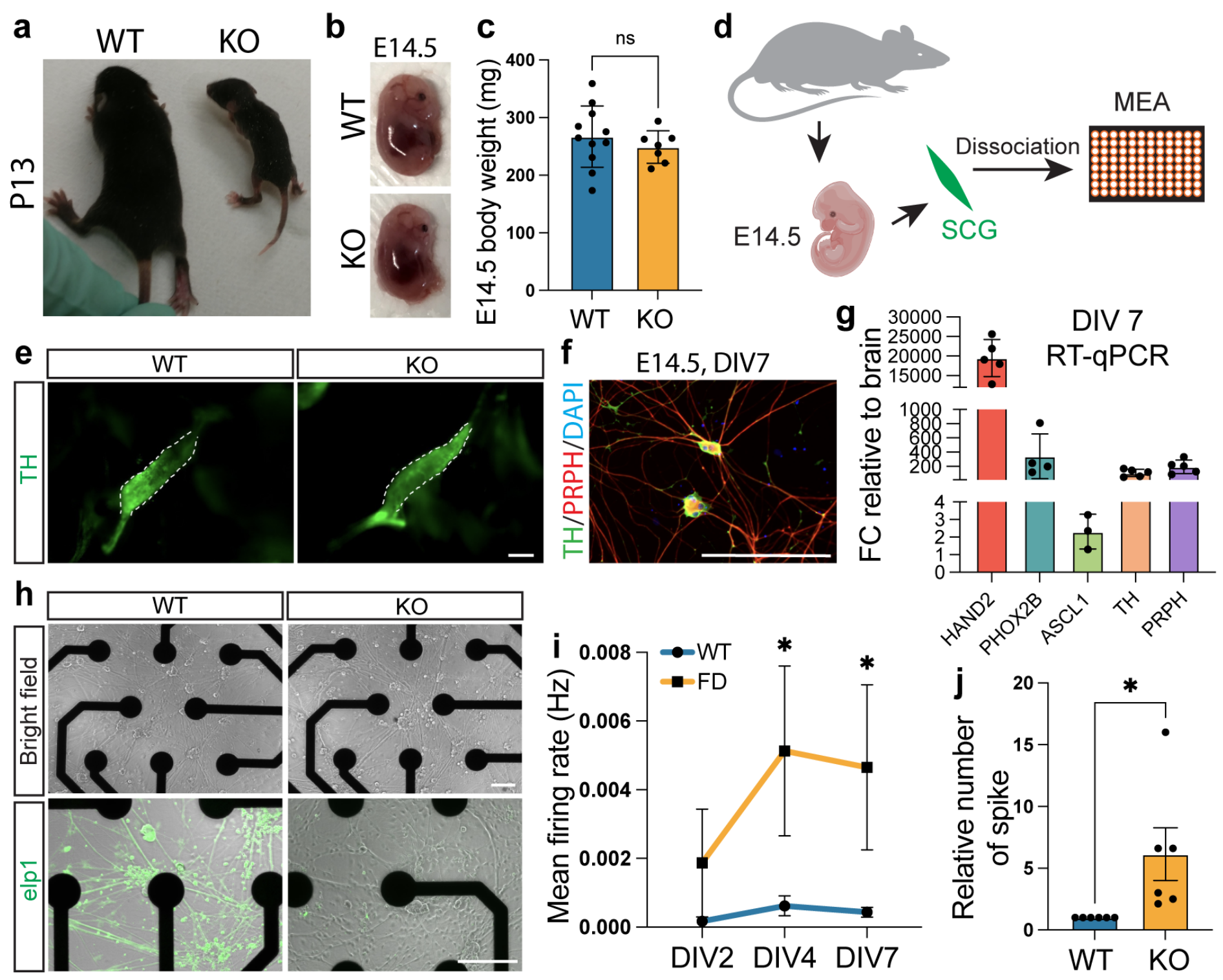

\section{Figure 3}

Sympathetic neuron hyperactivity in FD mouse models. (a) A survived P13 wnt1/Sox10cre;Elp1LoxP/LoxP-CKO mouse, has reduced body size. (b-c) E14.5 CKO embryos have no difference in appearance (b) as well as in body weight (c). n=7-11 biological replicates. Two-tailed Student's t-test. Error bar represents the SEM. (d) Schematic illustration of SCG neuron culture and experimental design. (e) Whole mount immunostaining of TH+ SCGs shows that E14.5 CKO SCGs have similar size and shape compared to control. (f) Immunostaining shows TH+ (green)/PRPH+ (red) neurons in culture after one week. (g) RT-qPCR shows symN gene expressions on DIV7. $n=3-5$ biological replicates. Error bar represents the SEM. (h) Dissociated SCG neurons on MEA plates show similar neural morphology and distribution at lower magnitude (top row). Immunostaining of Elp1 (bottom row) confirms elp1 depletion in CKO SCGs. (i) SCG neuron MEA recording in time course shows CKO symN hyperactivity. $n=3-8$ biological replicates. Two-way ANOVA followed by Šídák multiple comparisons. Error bar represents the 
SEM. ${ }^{*} p<0.05$. (j) Relative spike number on DIV7 is quantified by MEA. $n=6$ biological replicates. Twotailed Student's t-test. Error bar represents the SEM. * $p<0.05$. Scale bars represent $200 \mu \mathrm{m}$. See also Sup. Fig. 6.

\section{Fig 4. Hyperactivity is caused by NE trafficking defects}
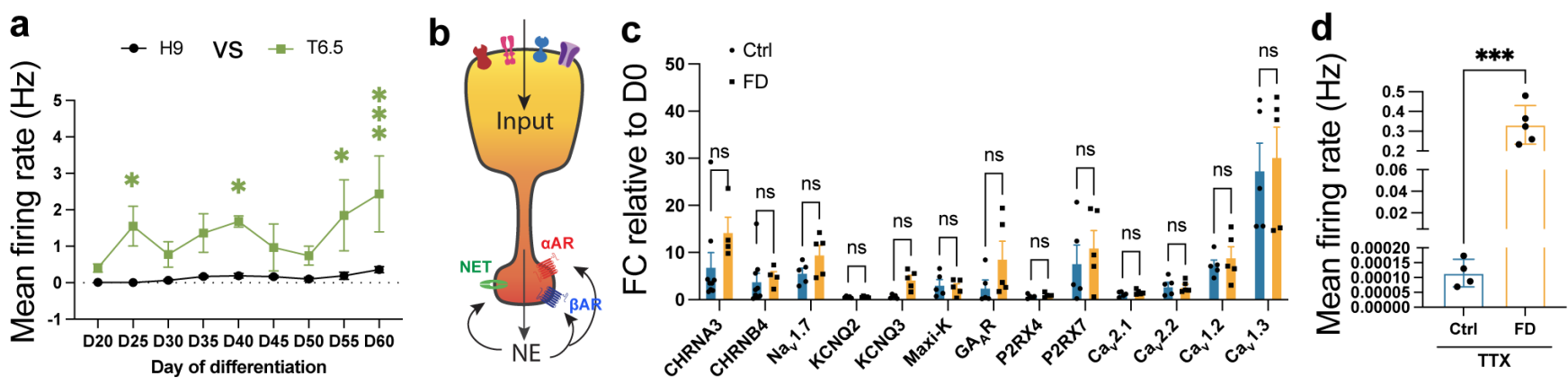

e

f
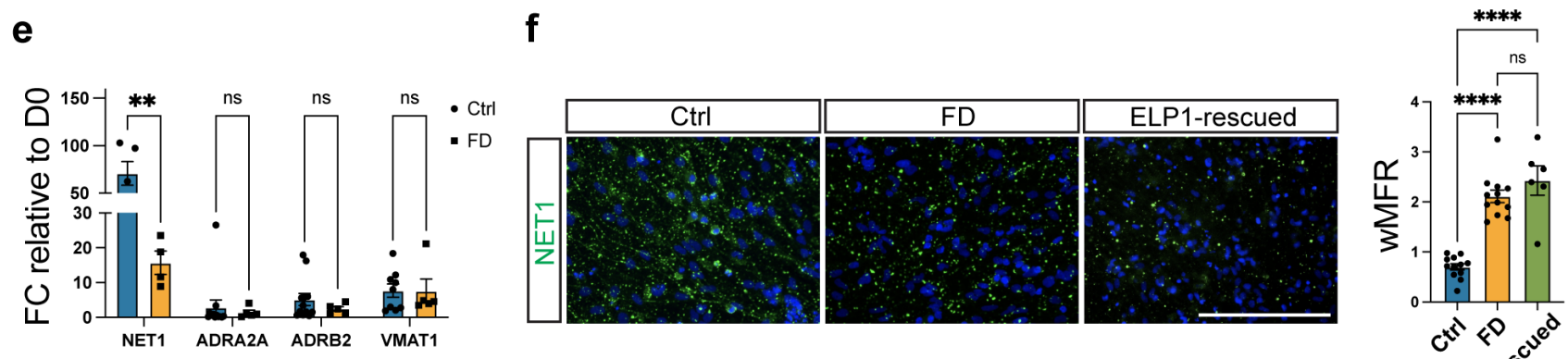

g

h

i

Intracellular NE
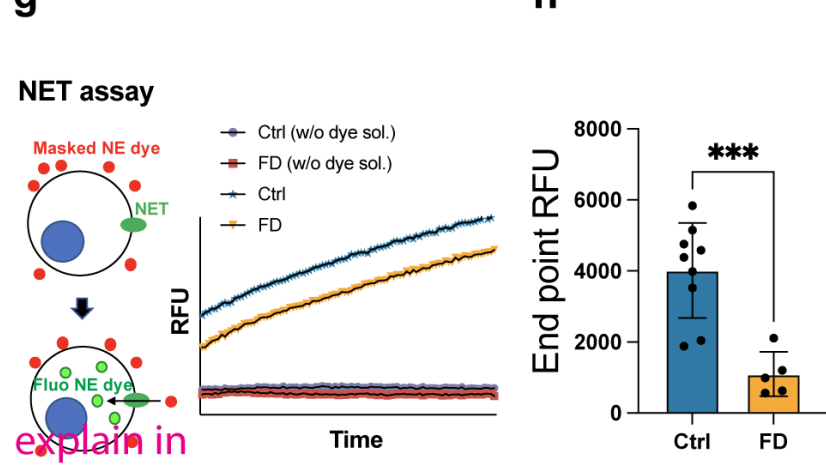

figure legend

k
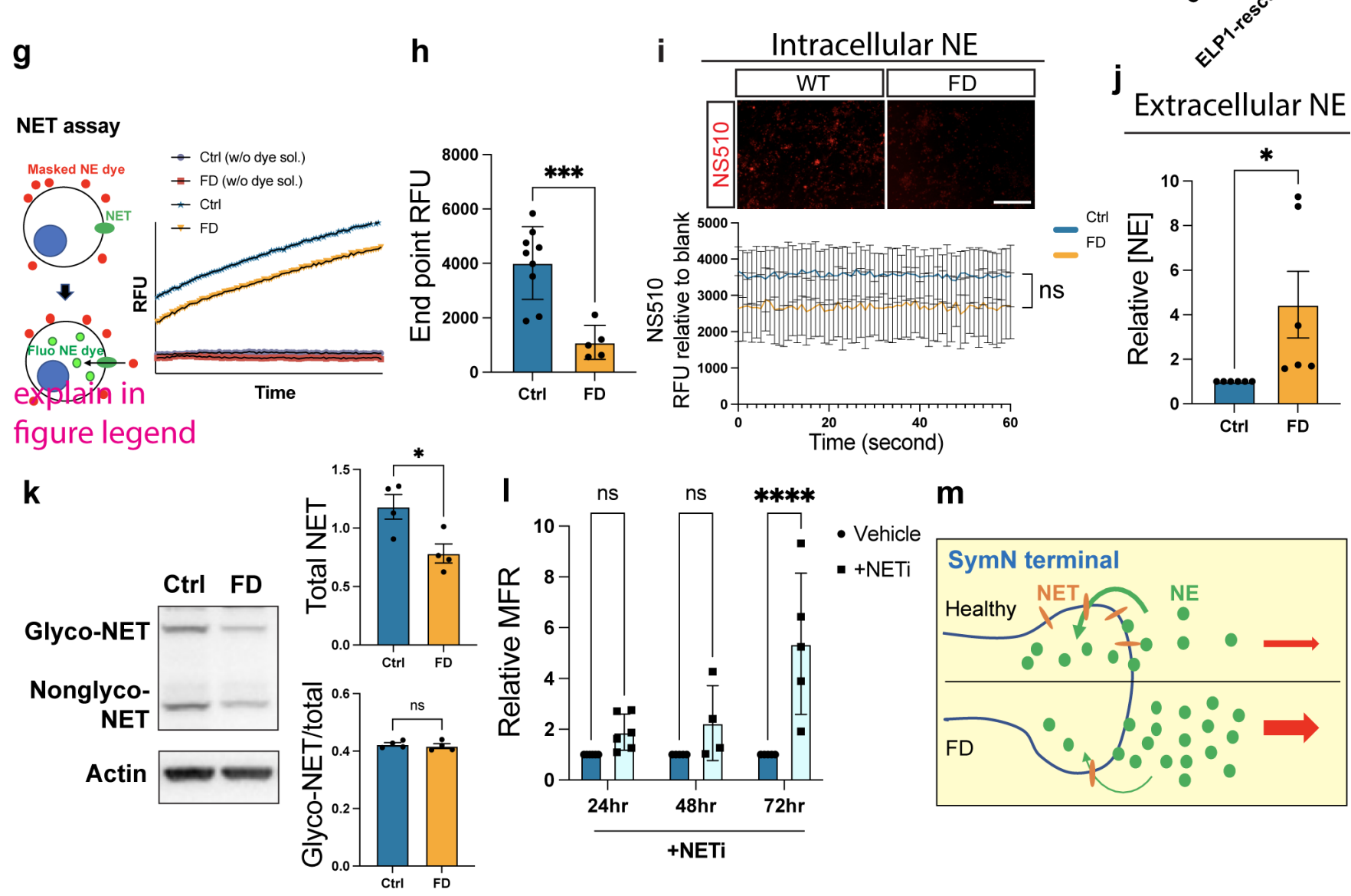

m

\section{Figure 4}

Reduced NE reuptake caused by NET deficiency leads to FD symN hyperactivity. (a) MEA measurements on symNs derived from iPSC-rescued-T6.5 still shows hyperactivity compared to hESC-ctl-H9 (same data 
as in Fig. $2 \mathrm{~b}$ for $\mathrm{ctl}$ ). $\mathrm{n}=4-9$ biological replicates. Two-way ANOVA followed by Šídák multiple comparisons. Error bar represents the SEM. ${ }^{*} p<0.05$, ${ }^{\star *} \mathrm{P}<<0.001$. (b) Cartoon representing the rationale of the screening of ion channels/receptors/transporter that may contribute to electric activity changes in symNs. (c) Screening by RT-qPCR shows no difference in expressions of ion channels/receptors receiving presynaptic signals between control and FD. $n=5$ biological replicates. Multiple unpaired Student's t-test. Error bar represents the SEM. (d) MEA assay still shows hyperactive FD symNs after treatment with 100 $\mu \mathrm{M}$ TTX. $\mathrm{n}=4-5$ biological replicates. Two-tailed Student's t-test. Error bar represents the SEM. ${ }^{\star * *} \mathrm{P}<0.001$. (e) RT-qPCR shows decreased NET RNA levels in FD symNs. $n=4-9$ biological replicates. Multiple unpaired Student's t-test. Error bar represents the SEM. ${ }^{*} \mathrm{P}<0.01$. (f) Immunostaining for NET supports decreased NET levels in FD symNs (left). MEA shows increased weighted mean firing rate in FD and ELP1-rescued symNs (right). $n=6-12$ biological replicates. One-way ANOVA followed by Tukey's multiple comparisons. ${ }^{* \star * *} \mathrm{P}<0.0001$. (g) Cartoon (left) illustrates the principle of NET reuptake assay. An NE simulating fluorophore is incubated with symNs in a fluorescence-masking reagent, which blocks the fluorescent signals (red dots outside of symNs). Only when the fluorophore is taken up by NET, the intracellular GFP signals can be detected (green dots inside of symNs). The chart (right) shows the trend of NE reuptake efficiency between control and FD symNs over time. (h) Quantification of the end point GFP intensities between control and FD symNs. n=5-9 biological replicates. Two-tailed Student's t-test. Error bar represents the SEM. ${ }^{*} * P_{0}<0.001$. (i) NE imaging using NS510 shows lower intracellular NE in FD symNs. Images in top row show representative levels of NE in symNs. ELISA-based time lapse recording in bottom row shows FD symNs has lower but nonsignificant NE constantly. $n=4$ biological replicates. Twoway ANOVA followed by Šídák multiple comparisons. Error bar represents the SEM. (j) ELISA assay shows higher secreted NE in FD symNs. $n=6$ biological replicates. Two-tailed Student's t-test. Error bar represents the SEM. ${ }^{*} \mathrm{P}<0.5$. ( $\mathrm{k}$ ) Western blot analysis of NET glycosylation. $\mathrm{n}=4$ biological replicates. Two-tailed Student's t-test. Error bar represents the SEM. ${ }^{*} \mathrm{P}<0.5$. (I) Healthy control symNs are treated with NETi $(2 \mu \mathrm{M})$ and electric activity is measured by MEA overtime. Neural activity gradually increases after the treatment. $n=4-6$ biological replicates. Two-way ANOVA followed by Šídák multiple comparisons. Error bar represents the SEM. $* \star \star * P<0.0001$. $(\mathrm{m})$ Cartoon of working hypothesis, in which the downregulated NET level in FD symNs leads to over-released NE, and increases symN activity. In a, c, d, e, $\mathrm{f}, \mathrm{g}, \mathrm{h}, \mathrm{l}, \mathrm{j}, \mathrm{k}, \mathrm{I}$ data from hESC-ctl-H9 and iPSC-ctl-C1.2 are pooled as control; in c, d, e, f, g, h, l, j, k, data from iPSC-FD-S2.3 and iPSC-FD-S3 are pooled as FD. Scale bars represent $200 \mu \mathrm{m}$. 
Fig 5. Mini drug screen to rescue symN hyperactivity in FD
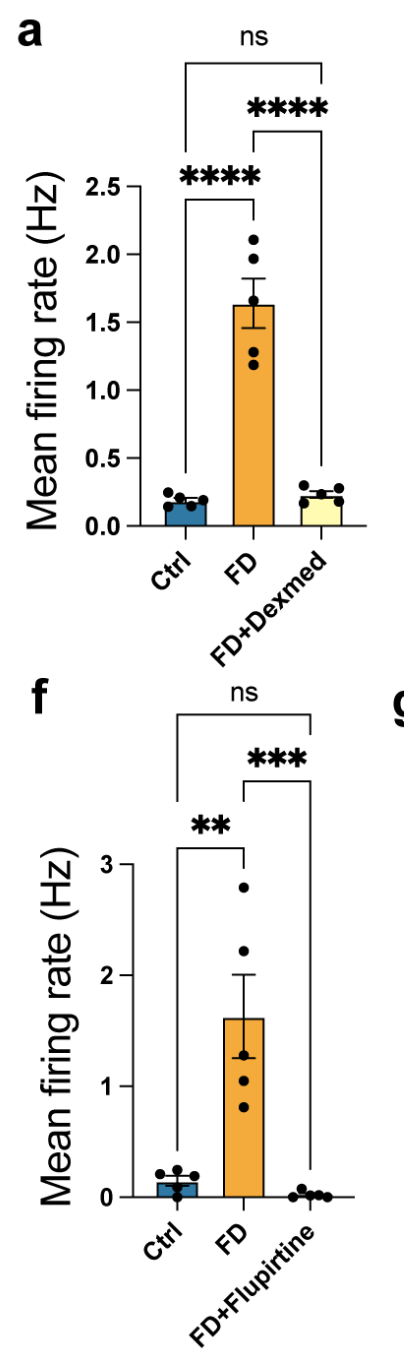
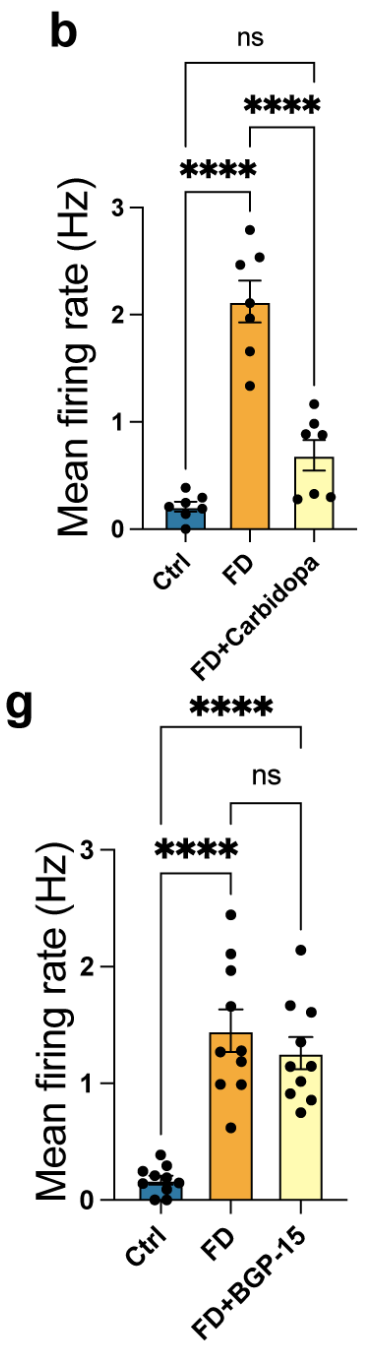
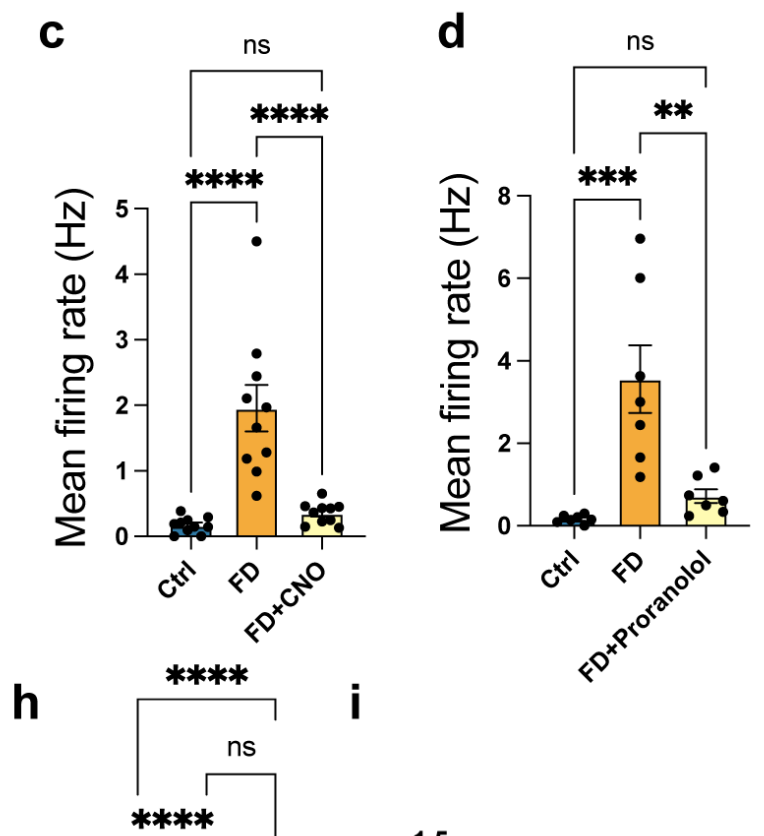

e
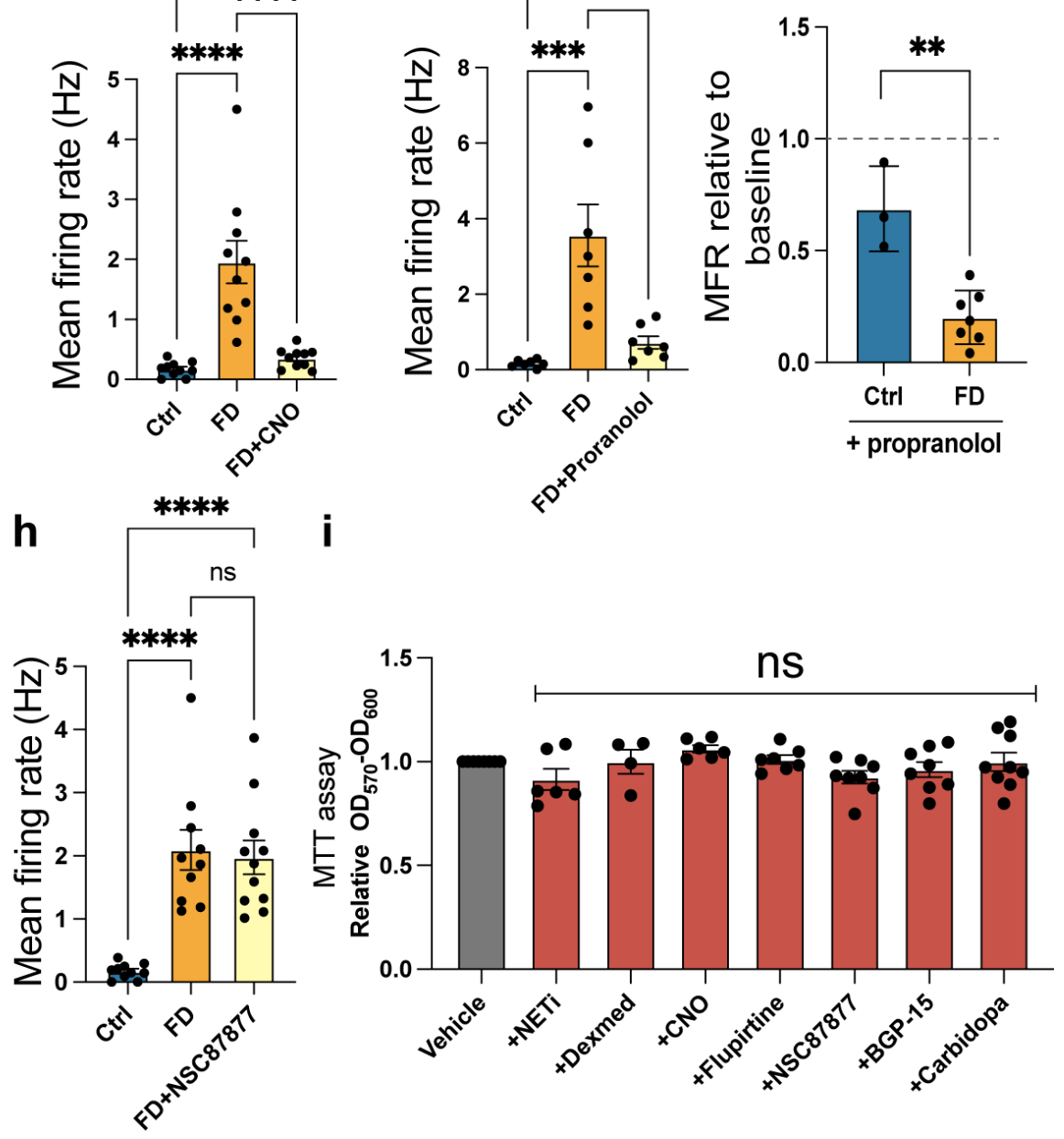

Figure 5

Mini drug screen to rescue symN hyperactivity in FD. A selection of drugs are added to FD symN on day 35, followed by MEA measurements. The dosage and treating conditions of each are: (a) Dexmed (60 $\mu \mathrm{M})$ for 5 minutes. $n=5$ biological replicates. One-way ANOVA followed by Tukey's multiple comparisons. Error bar represents the SEM. ${ }^{\star \star \star \star} P<0.0001$. (b) Carbidopa $(25 \mu \mathrm{M})$ for 5 minutes. $n=7$ biological replicates. One-way ANOVA followed by Tukey's multiple comparisons. Error bar represents the SEM. $\star \star \star \star P<0.0001$. (c) Clozapine ( $3 \mu \mathrm{M})$ for up to 72 hours in order to stimulate NET expression. $n=9-10$ biological replicates. One-way ANOVA followed by Tukey's multiple comparisons. Error bar represents the SEM. ${ }^{* \star \star *} P<0.0001$. (d) Propranolol $(1 \mu \mathrm{M})$ for 5 minutes. $n=7$ biological replicates. One-way ANOVA followed by Tukey's multiple comparisons. Error bar represents the SEM. ${ }^{*} P<0.01,{ }^{*} * P<0.001$. (e) Shows the differing ratio of firing after propranolol treatment of both ctrl and FD neurons. $n=3-7$ biological replicates. Two-tailed Student's t-test. Error bar represents the SEM. ${ }^{*} \mathrm{P}<0.01$. (f) Flupirtine $(15 \mu \mathrm{M})$ for 5 minutes. $n=5$ biological replicates. One-way ANOVA followed by Tukey's multiple comparisons. Error bar represents the SEM. ${ }^{\star *} P<0.01,{ }^{\star} * \star P<0.001$. (g) BGP-15 $(10 \mu \mathrm{M})$ for 5 minutes-72 hours. $n=10$ biological 
replicates. One-way ANOVA followed by Tukey's multiple comparisons. Error bar represents the SEM. (h) NSC87877 $(10 \mu \mathrm{M})$ for 5 minutes-72 hours. $n=10-11$ biological replicates. One-way ANOVA followed by Tukey's multiple comparisons. Error bar represents the SEM. The results are compared with those in untreated control and FD symNs. (i) The toxicity of all drugs used in this study (including NETi) is evaluated by MTT assay. $n=4-9$ biological replicates. One-way ANOVA followed by Tukey's multiple comparisons. Error bar represents the SEM. In a-h, data from hESC-ctl-H9 and iPSC-ctl-C1.2 are pooled as control; data from iPSC-FD-S2.3 and iPSC-FD-S3 are pooled as FD. See also Sup. Fig. 7
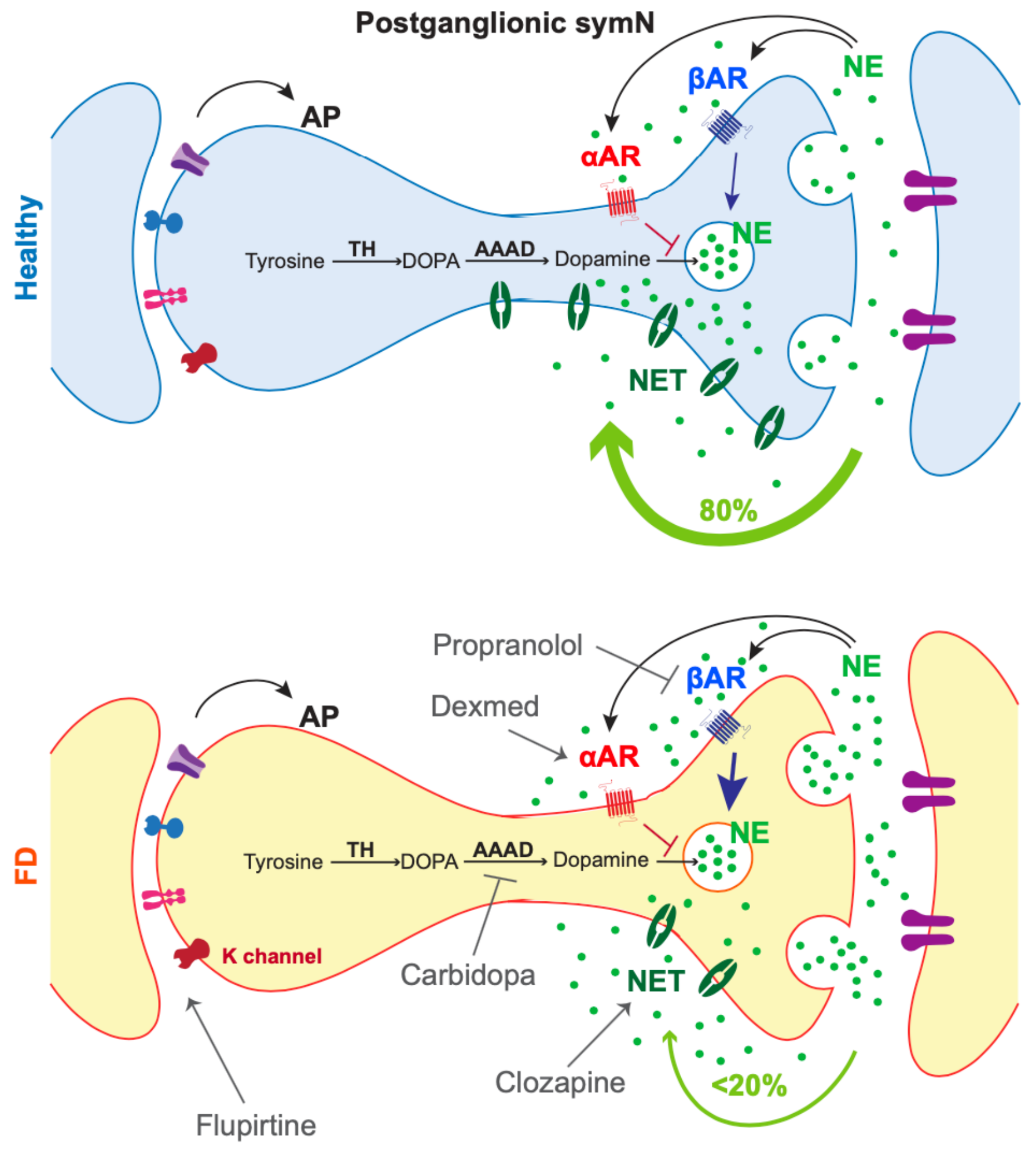


\section{Figure 6}

Model. In healthy postganglionic sympathetic neurons (symNs) (a), norepinephrine (NE) secretion in the axon terminal is properly regulated by norepinephrine transporter (NET), which reuptakes about $80 \%$ of released $\mathrm{NE}$, $a$ adrenergic receptors ( $\mathrm{aAR}$ ), which downregulate NE secretion and $\beta$ adrenergic receptors $(\beta A R)$, which upregulate NE secretion. In FD symNs (b), NET expression is decreased, leading to oversecreted NE. In addition, our results indicate that $\beta A R$ in FD symNs might have stronger response than healthy symNs, which might further strengthen the release of NE and symN activity. Several therapeutic targets are identified in this study using a selection of clinical compounds: flupirtine activates potassium channels ( $\mathrm{K}$ channel) and induces neural depolarization; carbidopa blocks NE synthetic pathway and thus reduces NE overspill; dexmedetomidine (Dexmed) activates aAR while propranolol inhibits $\beta A R$. All these drugs suppress FD symN hyperactivity in vitro.

\section{Supplementary Files}

This is a list of supplementary files associated with this preprint. Click to download.

- WuSupp.Figures.pdf

- SupplementaryFigurelegends.docx

- Table1.docx

- Table2.docx

- Supp.Vid1D7CM10x.mp4 